\title{
Flow Control by Slot Position and Noise Baffle in a Self-Recirculation Casing Treatment on an Axial Fan-Rotor
}

\author{
Xiangjun Li, ${ }^{1,2}$ Stephen Spence, ${ }^{2}$ Hua Chen, ${ }^{3}$ Wuli Chu, ${ }^{1}$ and Lee Gibson ${ }^{2}$ \\ ${ }^{1}$ School of Power and Energy, Northwestern Polytechnical University, Xi'an, China \\ ${ }^{2}$ School of Mechanical and Aerospace Engineering, Queen's University Belfast, Belfast, UK \\ ${ }^{3}$ National Laboratory of Engine Turbocharging Technology, Tianjin, China \\ Correspondence should be addressed to Wuli Chu; wlchu@nwpu.edu.cn
}

Received 11 August 2016; Revised 29 November 2016; Accepted 13 December 2016; Published 11 January 2017

Academic Editor: Jechin Han

Copyright (C) 2017 Xiangjun Li et al. This is an open access article distributed under the Creative Commons Attribution License, which permits unrestricted use, distribution, and reproduction in any medium, provided the original work is properly cited.

\begin{abstract}
To address the situations where the casing treatment needs to be used to stabilize axial compressors through strong recirculation, this paper initiated a CFD study to investigate how the flow could be suitably controlled in the casing treatment to minimize the efficiency penalty and increase the flow range. A counter-swirl self-recirculation casing treatment was first designed on a low speed axial fan rotor as a baseline case. Then three different slot positions and the influence of including the noise baffle were numerically studied. Based on the understanding of their coeffects, the shorter noise baffle was considered and it was found that the highest efficiency was achieved in the case of the upstream slot when the length of baffle was suitably adjusted to balance the incoming flow and recirculation. The largest flow range was achieved by locating the slot at the most downstream position and using a $50 \%$ length baffle since it suitably controlled the recirculating flow and relieved the separation at the low-span region. An optimization study showed that the optimum length of the baffle for efficiency was always larger than for the flow range. Both of the two optimum values reduce as the slot moves downstream.
\end{abstract}

\section{Introduction}

In aeroengine and other applications where a wider flow range is required, casing treatments are commonly employed on compressors for their cost-effectiveness in improving the system stability. The concept of casing treatment is not new and the earliest experiments date back to the 1960s when the NASA Lewis Research center conducted tests of bleeding and blowing through the casing for their influences on the stall range of a high-speed single stage axial-flow compressor [1]. In the decades since then, numerous research investigations have been conducted in this area. Different types of casing treatments were developed, tested, and applied. Although the initiation of the instability of compressors is still not fully understood, the results of previous studies have confirmed the effectiveness of casing treatments in controlling the onset of stall and system surge. Commonly, the improvement in flow range is accompanied by an efficiency penalty. The influences on the tip leakage vortex, span-wise blockage, and shock waves (in high-speed cases) are widely accepted as the reason why casing treatment can improve the stability of compressors.

The self-recirculation casing treatment is a type of casing treatment employed in both axial compressors and centrifugal compressors. The idea of "self-recirculation" initiated from the combination of bleeding and blowing, where the air is recirculated by bleeding from a downstream port and reinjecting it into the upstream flow field [2]. Different from the commonly used slot type [3] and groove type [4], the selfrecirculation casing treatment is designed with an isolated recirculation flow path which prevents the recirculation flow from directly interacting with the tip flow field of rotor. Therefore, the system has the potential to produce stronger recirculation; the bleed and injection can be more effectively controlled by utilizing the steam-wise pressure gradient.

The literature from previous studies shows that, when applied in axial-flow compressors, the amount of recirculation air was empirically minimized to less than $5 \%$ of 
overall mass flow rate due to its detrimental impact on the performance. The questions of where and how much to bleed and blow can be critical to the balance that is achieved between flow range improvement and efficiency penalty [2]. The work reported in previous papers by Hathaway $[5,6]$ employed a moderate speed axial fan-rotor to investigate how the bleed and injection contribute to the performance and how to determine suitable positions for each. Later studies focused in more detail on the understanding of the flow mechanisms present. Yang [7] studied how the leakage vortex and inlet flow angle were influenced by the bleed and injection. Another study [8] showed the influence of selfrecirculation casing treatment on the shock-vortex interaction. The recent research of Guinet et al. [9] employed a 1.5stage transonic test rig and a carefully designed recirculation duct to study the influence of the tip gap on the stall margin improvement and efficiency penalty of the casing treatment. A detailed parametric study of the recirculation duct was then conducted to investigate the interaction between casing flow and rotor tip flow [10].

In centrifugal compressors, the concept of self-recirculation is employed in the widely used "ported shroud" casing treatment [11]. Compared with the applications in axial-flow compressors, distinct differences can be found in centrifugal compressors where the casing treatment is typically designed to produce much higher levels of recirculation (even over $30 \%$ of the inlet flow) [12]. Additionally, because of the significant variation of positive or negative pressure differential along the shroud line of compressors, the casing treatment is designed to suck in flow near choke point while recirculating tip flow near the stall point to achieve a wider flow range, as explained by Fisher [13]. It is obvious that the position of the slot is the most critical parameter.

In the casing treatment of axial-flow compressors, the above-mentioned variation between suction and recirculation is seldom considered in design, which is probably because of their low blade load and strict limitation in recirculation mass flow rate and efficiency penalty. However, to consider the situations where the casing treatment needs to be used to stabilize compressors through strong recirculation, such as tip-critical compressors with significant SS separation or reverse flow, the control of recirculation should be taken into account for minimum detriment. The objective of this study is thus to identify the suitable method of controlling the recirculation flow for a larger flow range and minimum losses in axial compressors. In this paper, a numerical study of self-recirculation casing treatment was applied to a low speed axial fan-rotor. After a brief introduction of the CFD method in Section 2, a counterrotating self-recirculation casing treatment, which is typical in centrifugal compressors and able to strongly suck and inject air at different operating points, was designed and studied as a baseline case in Section 3. Then, to improve the efficiency and flow range of the rotor, the influence of the slot position and the noise baffle was further studied in Section 4.
TABLE 1: Blade elements of fan-rotor [14].

\begin{tabular}{lcccccc}
\hline Profile & $r / \mathrm{mm}$ & $\beta 1 /^{\circ}$ & $\beta 2 /^{\circ}$ & $\xi /^{\circ}$ & $\theta /^{\circ}$ & $S / c$ \\
\hline Hub & 127.00 & 34.900 & -18.800 & 8.000 & 53.500 & 0.611 \\
1 & 129.54 & 35.400 & -16.900 & 9.200 & 52.400 & 0.623 \\
2 & 158.24 & 40.900 & 2.200 & 21.600 & 38.600 & 0.761 \\
3 & 185.42 & 45.700 & 16.100 & 30.900 & 29.600 & 0.898 \\
4 & 190.50 & 46.100 & 17.500 & 31.800 & 28.600 & 0.917 \\
5 & 216.15 & 49.900 & 26.600 & 38.300 & 23.300 & 1.040 \\
6 & 246.38 & 53.500 & 36.600 & 45.000 & 17.000 & 1.180 \\
Shroud & 254.00 & 54.200 & 38.800 & 46.400 & 15.500 & 1.216 \\
\hline
\end{tabular}

\section{Rotor Geometry and CFD Method}

A low speed axial fan-rotor was chosen for this study, which follows the geometry used in reference [14]. The design speed of the rotor was $1500 \mathrm{rev} / \mathrm{min}$, with 27 blades, a $508 \mathrm{~mm}$ outer tip diameter, and a $1.2 \%$ span tip clearance. The geometry of the blade is given in Table 1. All the span-wise elements employed the $\mathrm{C} 4$ circular arc profile family and were designed with a blade loading coefficient of almost unity. According to the hotwire measurements of Kang et al. [15], the stall originated from the tip region of the rotor and the range of reverse flow occupied about $20 \%$ of the span near the tip end wall at the deep stall point, therefore indicating that the operation of this rotor near stall was "tip-critical."

The grid for the CFD study was generated using the NUMECA AutoGrid5 software, as shown in Figure 1. To predict the development of the tip leakage flow, the single passage domain contained 17 layers of grids in the tip clearance and extended axially to 4.5 axial chord lengths upstream of the tip leading edge. The outlet was located 2 axial chord lengths downstream of the trailing edge to predict the dissipation of vortex and shear flow after the blade. The overall grid number for a single blade passage was 650,000. The $y^{+}$value of the first grid layer was less than 2 .

The flow field of the rotor was calculated using the RANS method within ANSYS CFX. The inlet condition was defined as the total atmospheric pressure and temperature at standard conditions. The mass flow rate was specified at the outlet boundary. The convergence criterion was set to a value of $1 e-4$ for the RMS residual values. Considering the transient effects of stall and the complexity of the flow field near the stall point, more strict criteria were used here than in the previous publications $[12,16]$ to discern the predicted point of instability; that is,

(a) the simulation does not reach convergence;

(b) the perturbation of efficiency is larger than $0.2 \%$;

(c) the reverse flow exceeds $10 \%$ of the span from the tip end wall;

(d) the outlet static pressure begins to fall with decreasing mass flow rate.

The flow was defined as stall flow if one of the four listed criteria was reached. Then the nearest point with a larger mass flow rate was defined as the near stall point of the simulation. 


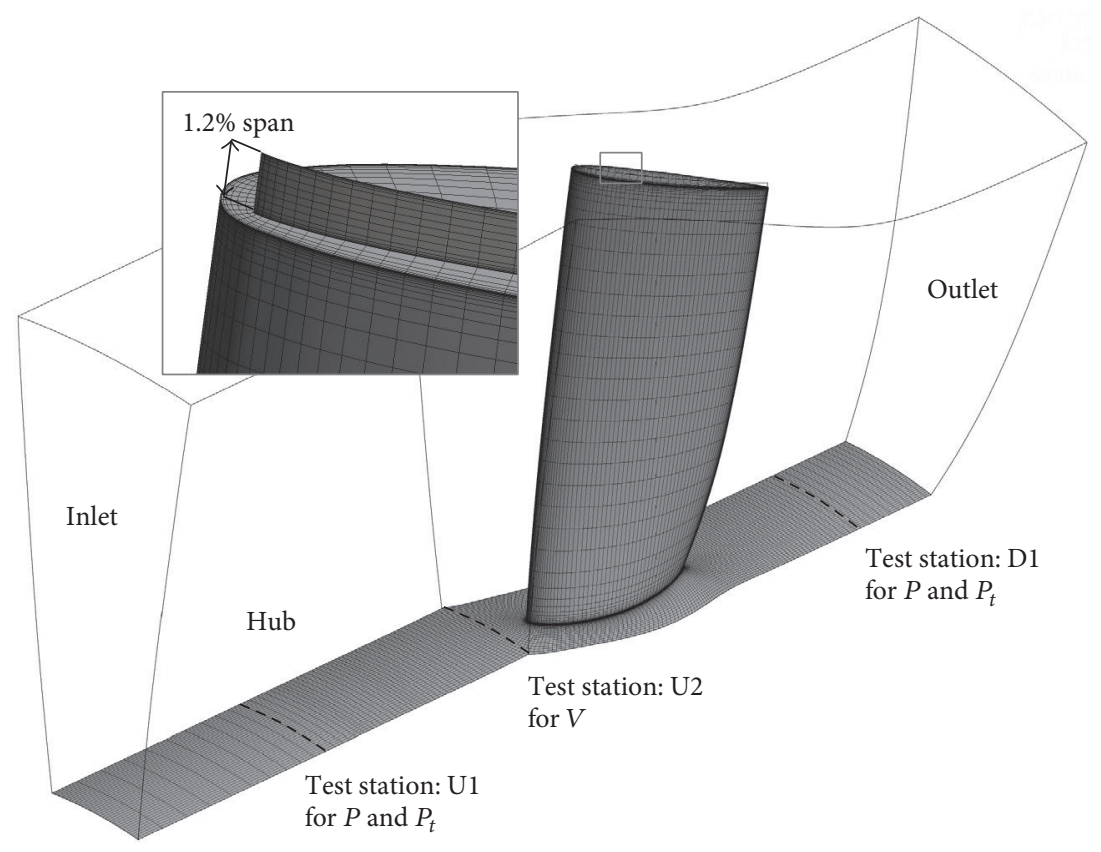

FIGURE 1: The computational grid developed for the rotor model.

TABLE 2: Grid independence study.

\begin{tabular}{lccccc}
\hline \multirow{2}{*}{ Grids } & Refine method & Number of meshes & \multicolumn{2}{c}{$m=0.61$} & \multicolumn{2}{c}{$m=0.33$} & \multicolumn{1}{c}{$\eta$} \\
\hline G1 & Baseline & 650,000 & 0.315 & 0.885 & 0.0852 \\
G2 & Span-wise & 917,000 & 0.304 & 0.889 & 0.0651 \\
G3 & Span-wise \& blade-to-blade & $1,443,000$ & 0.319 & 0.885 & 0.24 \\
\hline
\end{tabular}

Figure 2 compares the predicted performance with the experimental data presented by Kang et al. [15]. Note that although the near stall point is at $\phi=0.61$, the simulation results at lower mass flow rates are also presented for some cases that still reached convergence. The CFD study tested two types of turbulence model for their accuracy in predicting the separation flow; these were the SST model and $k-\omega$ model with automatic wall function. In Figure 2(a), the predicted total performance is in good agreement with the experimental data in the important NS point, except for the efficiency after stall. The reason may be connected to a discrepancy in the prediction of the axial flow between $20 \%$ and $50 \%$ span, which is influenced by the reverse flow in the tip region, as shown by the pitch-averages $V_{t}$ and $V_{a}$ in Figure 2(b). However, the characteristic shape of the experimental profiles, especially the axial and tangential component of reverse flow from $60 \%$ span to tip end wall, is accurately predicted. Compared with the SST turbulence model, the $k-\omega$ model predicted the outlet static pressure rise with higher accuracy, as shown in Figure 2(c). So the $k-\omega$ model was selected for the remainder of the study in this paper.

Table 2 provides the result of the grid independence study. The G1 grids were the baseline grids employed in the current simulation. The G2 grids were further refined in the span-wise direction and the overall number reached about 900,000. The G3 grids were refined in both the spanwise and the blade-to-blade directions, so the overall number reached about 1,440,000. According to Table 2, the only major difference between the three grids was in the predicted efficiency at $\phi=0.33$. However, because of the discrepancy in the prediction of the axial flow between $20 \%$ and $50 \%$ span, as shown in Figure 2, neither of the two refined grids predicted better result than the baseline mesh. Therefore, the solution of the Gl grids was judged as grid independent.

\section{Compressor Performance due to Counter- Swirl Self-Recirculation Casing Treatment}

3.1. The Geometry of Baseline Casing Treatment. Figure 3(a) shows the sketched geometry of the baseline counter-swirl casing treatment. The outer radius of the casing treatment was $40 \mathrm{~mm}$ larger than the rotor. The recirculation duct was designed with an inner ring in the bottom, 27 leaned guide vanes that were straight in the axial direction, and a circumferentially continuous slot covering the first $2 / 3 \mathrm{ca}$ of the rotor tip. The noise baffle was also considered because of its aerodynamic influence. A comparison of the computational meshes is presented in Figure 3(b). For the case 


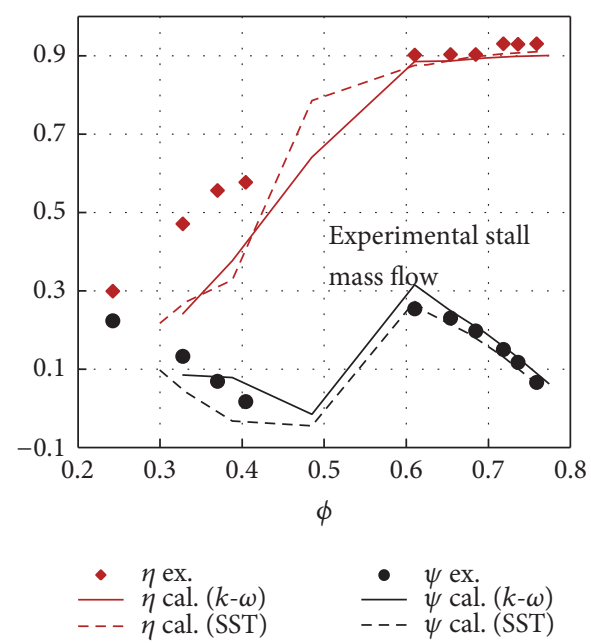

(a) Total performance
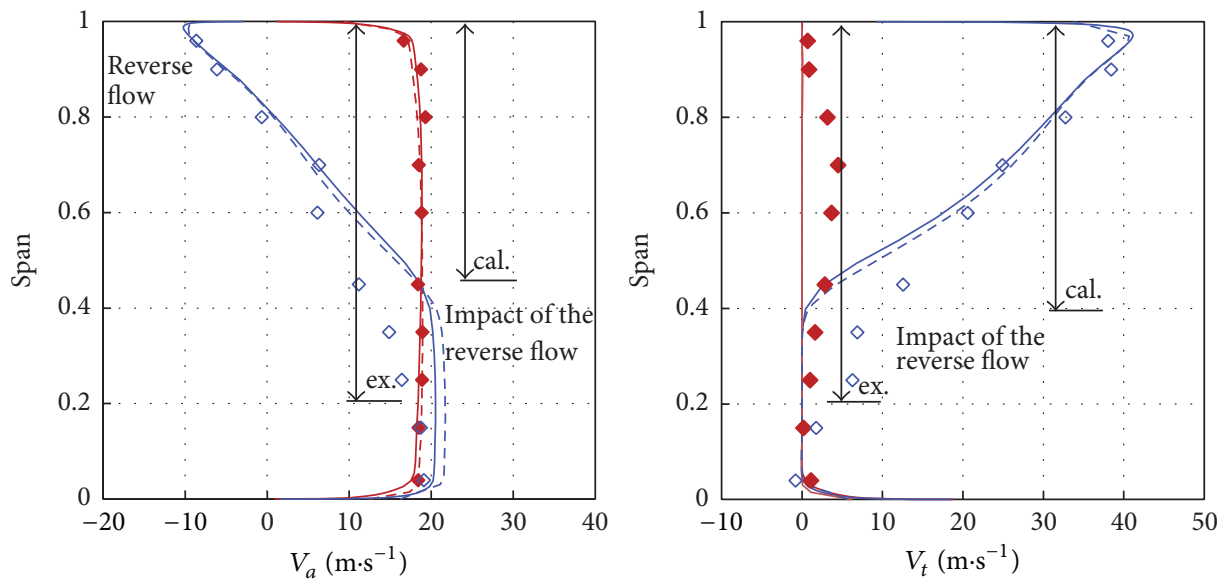

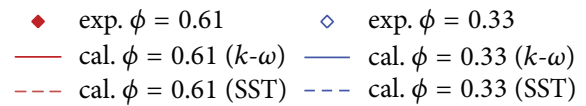

- $\exp . \phi=0.61 \quad \diamond \quad \exp . \phi=0.33$

— cal. $\phi=0.61(k-\omega)-$ cal. $\phi=0.33(k-\omega)$

- - c cal. $\phi=0.61$ (SST) - - cal. $\phi=0.33$ (SST)

(b) Inlet velocity (at test station U2)

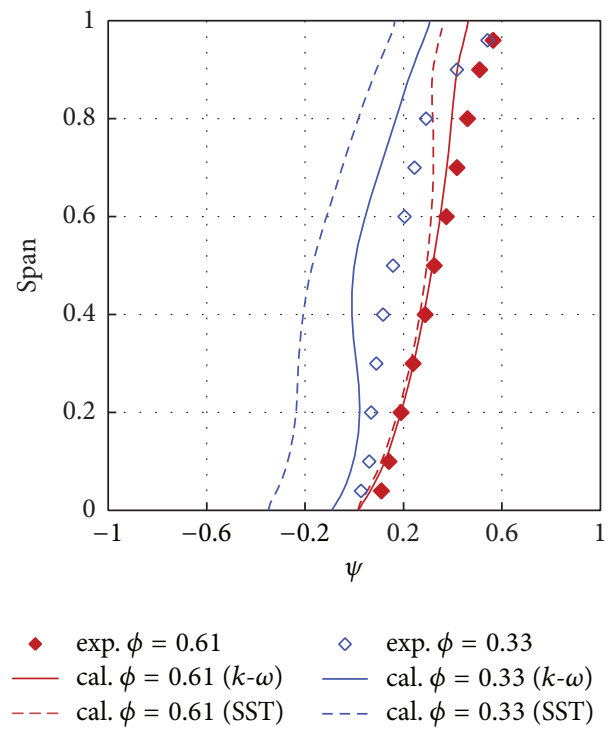

(c) Outlet static to total pressure rise coefficient (at test station D1)

FIgURE 2: Comparison between simulation results and experiment data [13]. 
A

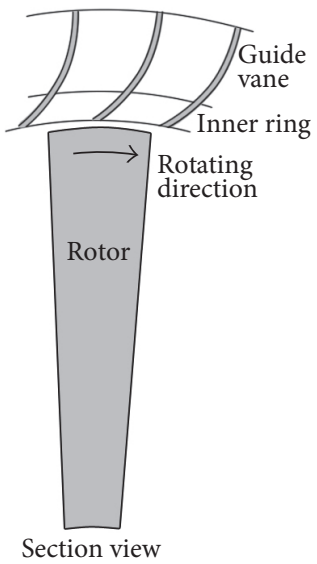

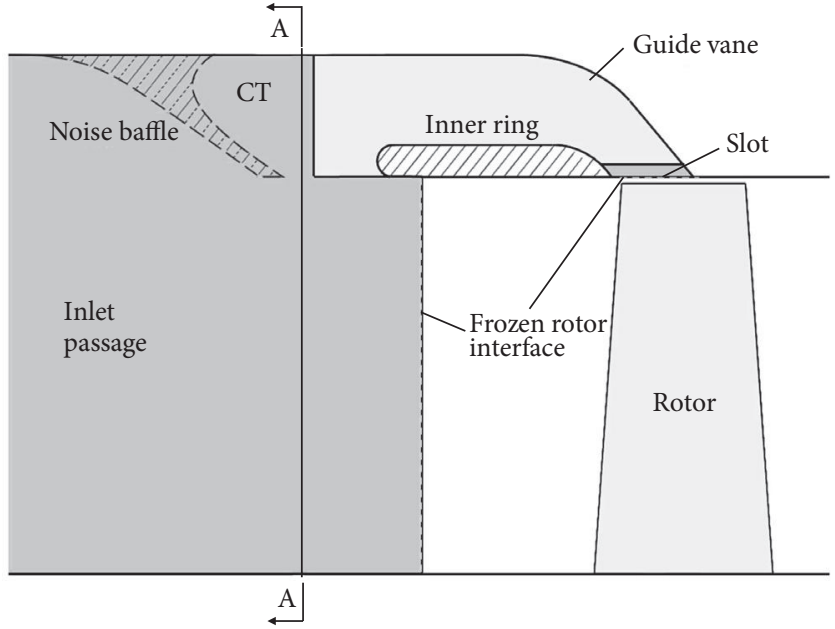

(a) Sketched view of casing treatment

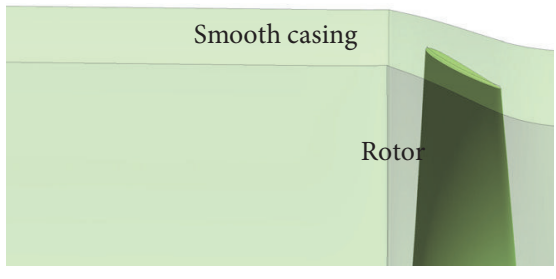

Baseline

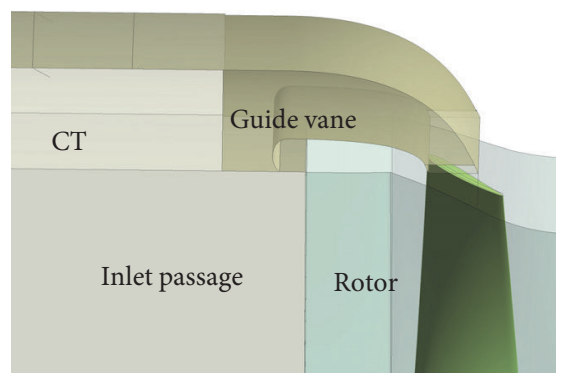

CT

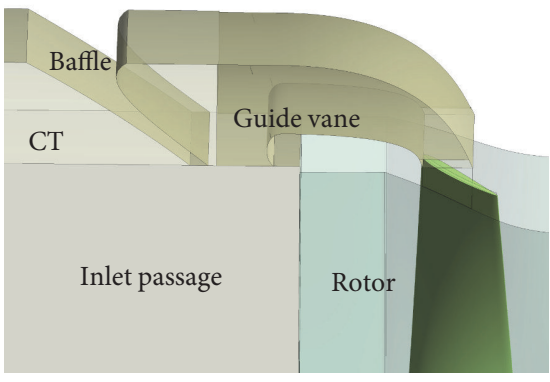

$\mathrm{CT}_{\text {baffled }}$

(b) Grids

FIGURE 3: Geometry and grids of the counter-swirl self-recirculation casing treatment.

with casing treatment (labeled with "CT"), the rotor uses the same grids as in the baseline rotor simulation except for a shorter inlet block in the rotor domain. The grids of the casing treatment were generated as an extension of the inlet passage by applying the same $y^{+}$criterion as was used in the rotor. Both of them were set as stationary domains and connected to the rotor domain using the Frozen Rotor interface. For the case with noise baffle (labeled with "CT $\mathrm{baffled}$ "), the cells included in the noise baffle block were excluded from the meshes thus forming the solid wall of the baffle. The stator domain extended axially to over $10.0 \mathrm{ca}$ upstream of the leading edge, because the extent of the recirculation could be significantly influenced by the duct when there is no noise baffle.

3.2. Influence of Recirculation Flow in Baseline Cases. Figure 4 shows the simulated total performance of the smooth wall casing (SW), nonbaffled casing treatment (CT), and baffled casing treatment $\left(\mathrm{CT}_{\text {baffled }}\right)$ in the stable flow range. In this figure, $m=4.2 \mathrm{~kg} / \mathrm{s}$ corresponds to $\phi=0.61$ and $m=$ $3.4 \mathrm{~kg} / \mathrm{s}$ corresponds to $\phi=0.34$. Compared with the simulation in Section 2, the longer inlet passage generated a thicker annular boundary layer at inlet to the blades and thus reduced the peak efficiency and flow range of the fan-rotor by comparison with the previous short inlet case. The flow range improvement was evaluated using the following equation:

$$
\Delta m=\left(\frac{m_{\mathrm{NS}, \mathrm{SW}}}{m_{\mathrm{NS}, \mathrm{CT}}}-1\right) \times 100 \% \text {. }
$$

It can be seen that both casing treatments increased the mass flow range by reducing the mass flow rate for the near stall point. $\mathrm{CT}_{\text {baffeld }}$ achieved a greater $\Delta m(19.2 \%)$ than CT (15.4\%), but both of them decreased the peak efficiency by about $6.5 \%$.

The reason for the flow range improvement can be seen in Figure 5 which shows the flow field at $m=3.4 \mathrm{~kg} / \mathrm{s}$, where the simulation of the SW case is judged as stalled according to the listed criteria (thus not shown on the map) even though it still reached convergence. Figure 5 shows the pitch-wise averaged axial velocity at the leading edge test station (U2, indicated in Figure 1). The simulated streamlines are also illustrated for each geometry case. Two contours of $V_{a}$ in the recirculation duct indicate the flow direction: red color means positive flow and blue means reverse flow (recirculation). At $m=3.4 \mathrm{~kg} / \mathrm{s}$, CT and $\mathrm{CT}_{\text {baffled }}$ both showed a similar impact 

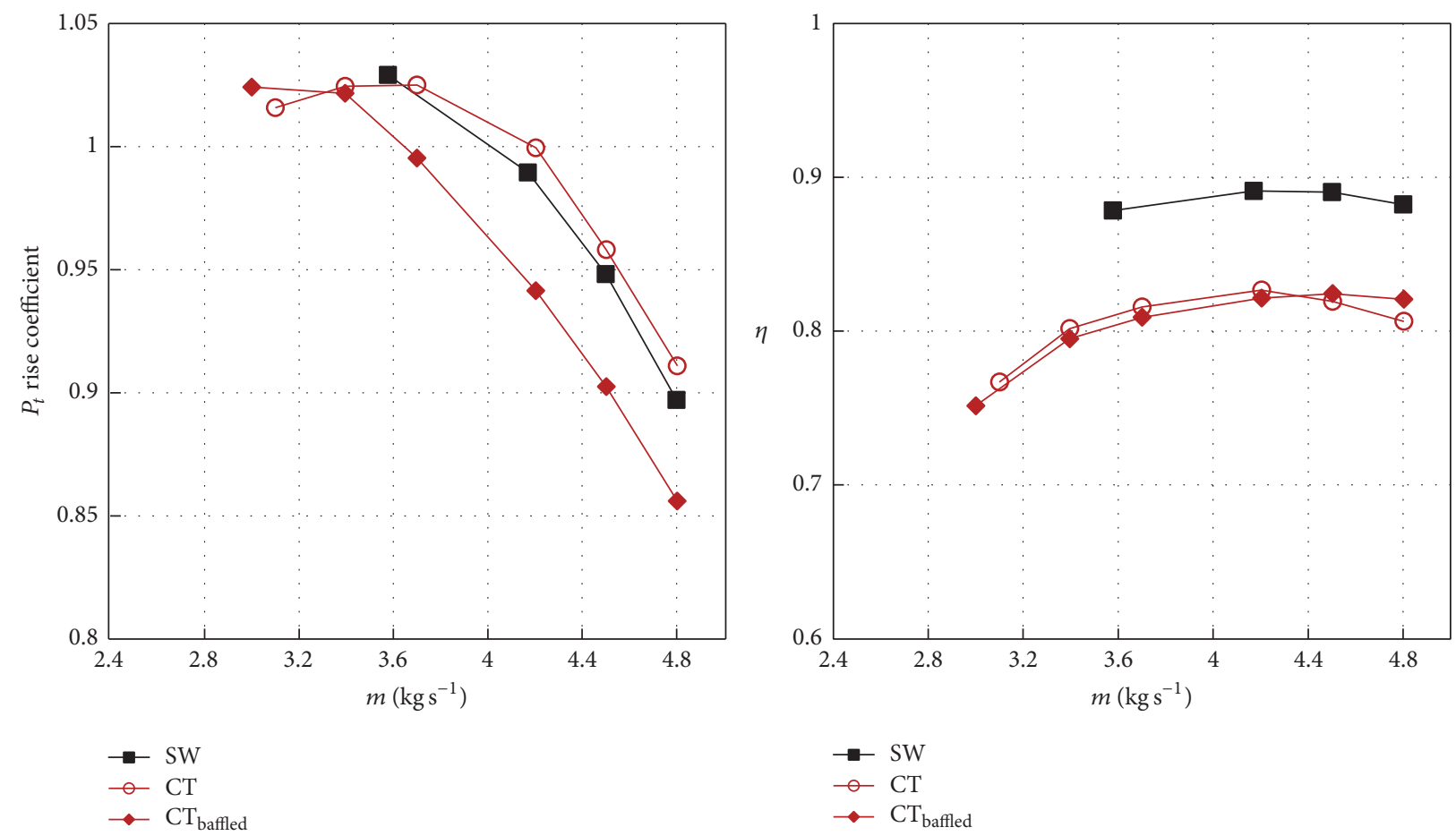

FIGURE 4: Overall performance of the baseline casing treatments.
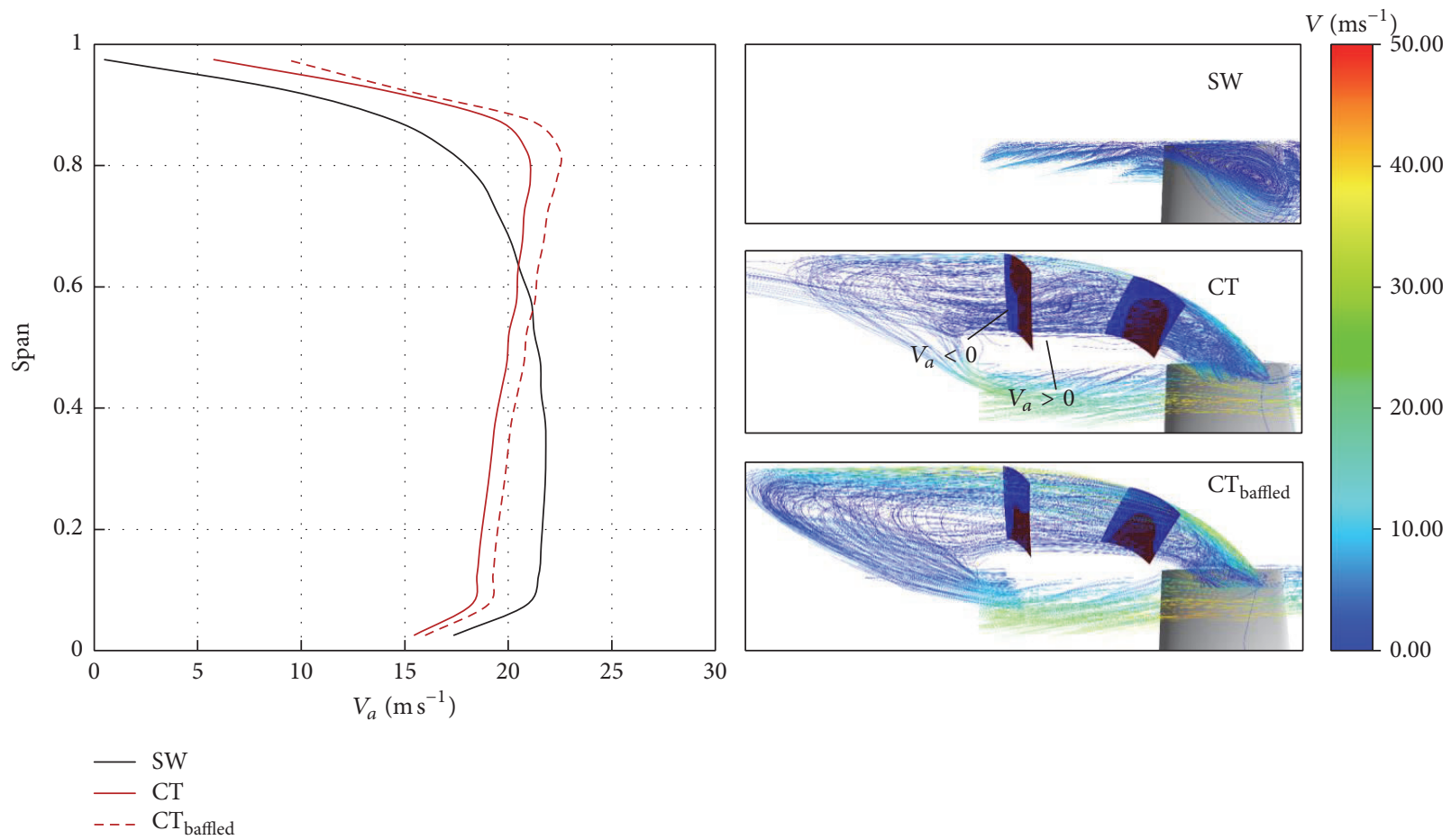

FIGURE 5: Inlet axial velocity at $m=3.4 \mathrm{~kg} / \mathrm{s}$ and the corresponding three-dimensional flow field.

on the flow field. The recirculation flow in both cases removed the significant tip blockage that was present in the SW case. The noise baffle was found to intensify the recirculation in the duct, as shown by the $V_{a}$ contours and streamlines. The tip region of the baffled casing treatment thus possessed higher axial momentum than the nonbaffled casing treatment, as evident in the span-wise plot of $V_{a}$.

The reduction of the peak efficiency is a more complex matter because efficiency is influenced by both work and loss. A quantitative analysis is given here to judge which of the two 


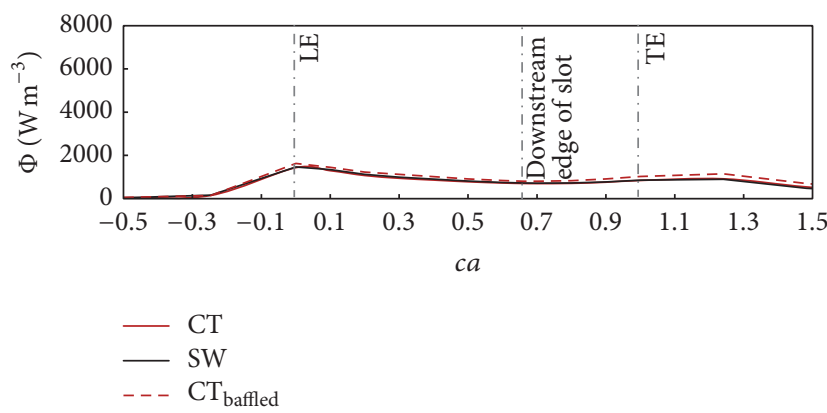

(a) Loss generated below $80 \%$ span

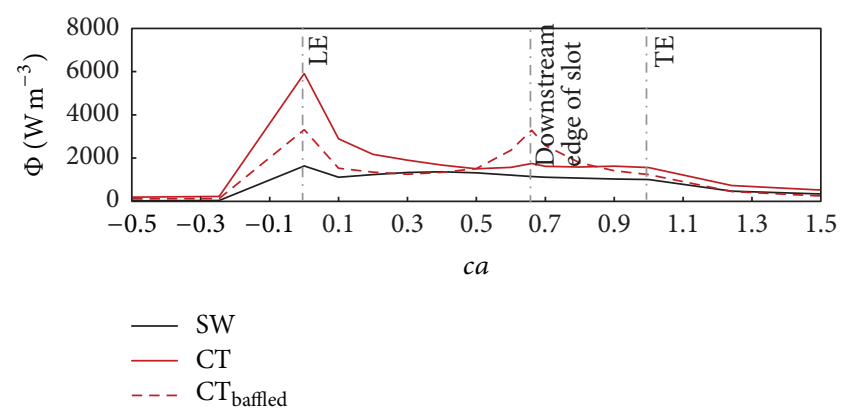

(b) Loss generated from $80 \%$ span to the casing

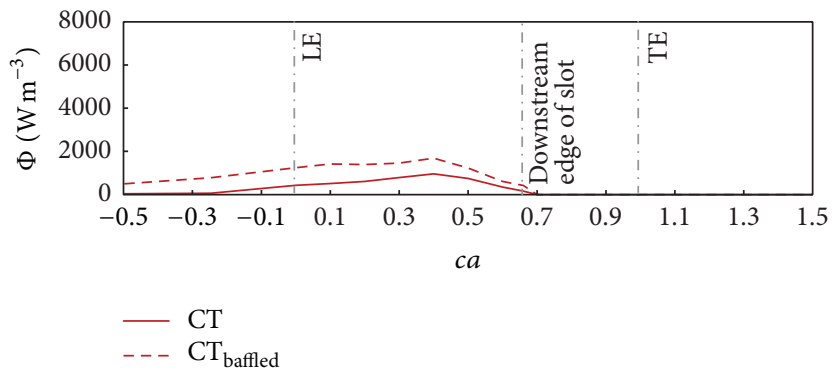

(c) Loss generated in the recirculation duct

FIGURE 6: Stream-wise distribution of loss generation at peak efficiency point ( $m=4.2 \mathrm{~kg} / \mathrm{s})$.

factors makes the major contribution. The definition of the isentropic efficiency is

$$
\eta_{\mathrm{SW}}=\frac{\pi^{(\gamma-1) / \gamma}-1}{\tau-1}
$$

According to its physical interpretation, the impact of recirculation should change both $\pi$ and $\tau$. Assume total temperature ratio becomes $\tau f_{w}$ and the total pressure ratio is changed to $\pi\left(f_{w} f_{l}\right)^{\gamma /(\gamma-1)}$, where $f_{w}$ and $f_{l}$ correspond to the change of input of work and loss. Then the efficiency can be expressed as

$$
\eta_{\mathrm{CT}}=\frac{\pi^{(\gamma-1) / \gamma} f_{w} f_{l}-1}{\tau f_{w}-1} .
$$

The 1st-order difference of $\eta_{\mathrm{CT}}$ is thus given by

$$
\begin{aligned}
d \eta_{\text {СТ }} & \\
= & \frac{\pi^{(\gamma-1) / \gamma} f_{w}}{\tau f_{w}-1} d f_{l} \\
& +\frac{\pi^{(\gamma-1) / \gamma} f_{l}\left(\tau f_{w}-1\right)-\left(\pi^{(\gamma-1) / \gamma} f_{w} f_{l}-1\right) \tau}{\left(\tau f_{w}-1\right)^{2}} d f_{w} .
\end{aligned}
$$

The values of $f_{w}$ and $f_{l}$ were calculated from the simulation results for the $\mathrm{SW}, \mathrm{CT}$, and $\mathrm{CT}_{\text {baffled }}$ cases. $d \eta_{\mathrm{CT}}$ was evaluated using $\eta_{\mathrm{CT}}-\eta_{\mathrm{SW}}$. Then the contribution of $f_{w}$ and $f_{l}$ could be estimated, as shown in Table 3.

According to Table 3, at peak efficiency point, the growing loss made a major contribution to the drop of efficiency in both the CT and the $\mathrm{CT}_{\text {baffled }}$ cases.
The distribution of loss sources was determined using the dissipation function $(\Phi)$. The physical interpretation of this function is the production of loss in specific time and volume. The stream-wise area-averaged dissipation function $\left(\Phi_{\text {area }}\right)$ for SW, CT, and $C_{\text {baffled }}$ is plotted in Figure 6 and the corresponding flow fields are shown in Figure 7. The loss generated from hub to $80 \%$ span was almost unaffected by the flow through the casing treatment, as shown by Figure 6(a). Illustrated by the bleed (red arrow) and injection (blue arrow) flows through the slot in Figures 7(b) and 7(c), the interaction between the duct flow and the main flow blocked the tip region in both the $\mathrm{CT}$ and $\mathrm{CT}_{\text {baffled }}$ cases. The shear flow around the leading edges was thus intensified close to the PS (labeled (i) in Figures 7(b) and 7(c)) and significantly increased the $\Phi_{\text {area }}$ between $80 \%$ span and $100 \%$ span, as shown by Figure 6(b). In the flow field of CT, the injection of duct flow separated the leakage vortex into two branches and induced a new tangential-axial swirling flow in the rear passage (labeled (ii)). For $\mathrm{CT}_{\text {baffled }}$, the interaction between duct flow and tip flow in the rear edge of the slot caused strong shear flow (labeled (iii)) and high dissipation in Figure 6(b). Additionally, the dissipation in the recirculation duct also contributed to the overall increase of losses, as shown by Figure 6(c). The stronger recirculation in $\mathrm{CT}_{\text {baffled }}$ generated higher loss in the duct than for CT.

It can be seen that the additional flow range and the reduction of peak efficiency are mainly associated with the interaction between the duct flow and tip flow. Therefore, in the next section, a series of numerical simulations will be applied to the $\mathrm{CT}$ and $\mathrm{CT}_{\text {baffled }}$ cases in order to investigate the possibilities for increasing the peak efficiency and flow range 
TABLE 3: The contribution of change of loss and work input to efficiency.

\begin{tabular}{lcccc}
\hline & \multicolumn{2}{c}{} & \multicolumn{2}{c}{$\Delta \eta$ due to input of work } \\
& Value & Contribution in percentage & Value & Contribution in percentage \\
\hline $\mathrm{CT}$ & -0.0703 & $116.7 \%$ & 0.0101 & $-16.7 \%$ \\
$\mathrm{CT}_{\text {baffled }}$ & -0.0658 & $98.5 \%$ & -0.0001 & $1.5 \%$ \\
\hline
\end{tabular}
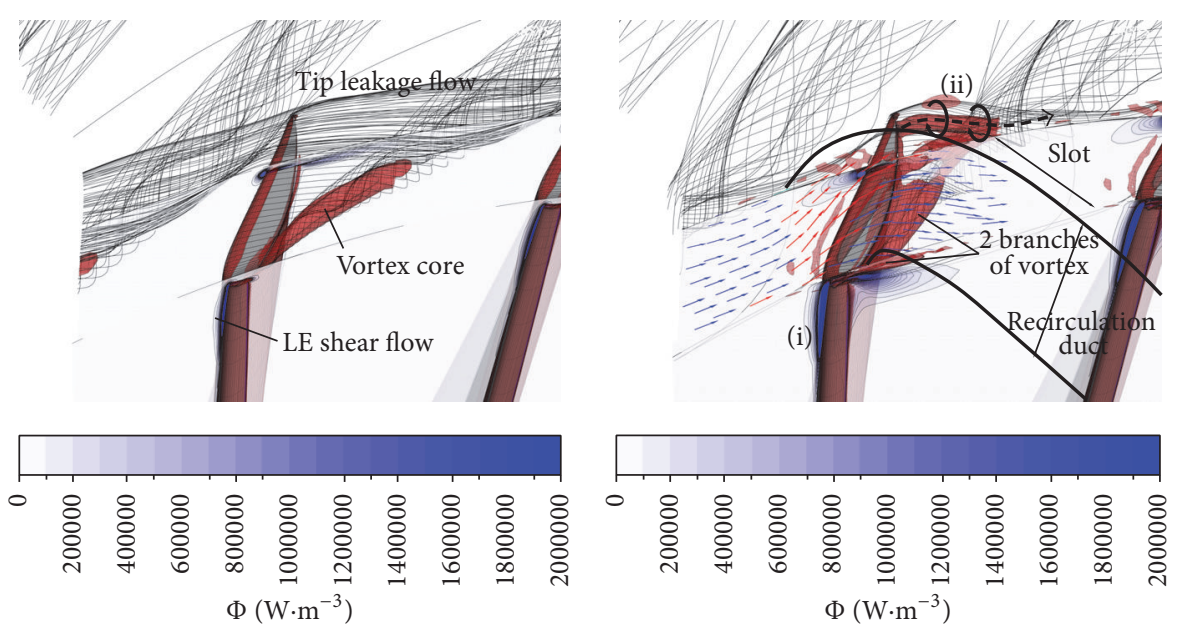

(a) $\mathrm{SW}$

(b) $\mathrm{CT}$

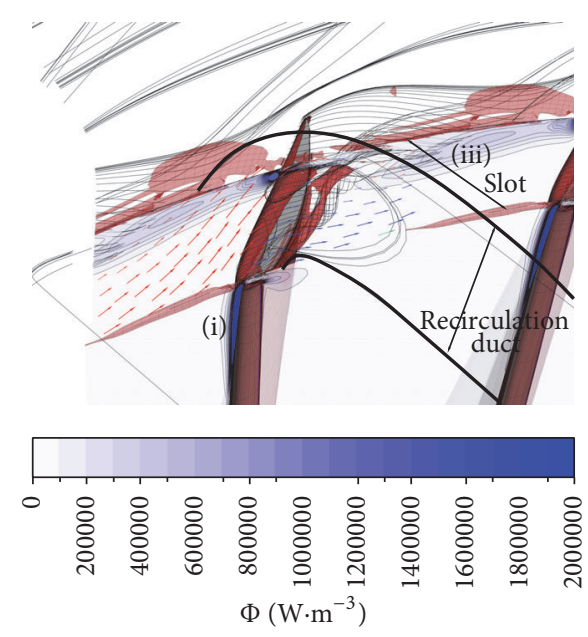

(c) $\mathrm{CT}_{\text {baffle }}$

FIGURE 7: The tip flow field at peak efficiency point $(m=4.2 \mathrm{~kg} / \mathrm{s})$.

by suitably controlling the interaction between the duct flow and tip flow field.

\section{Improvement of Rotor Performance by Duct Flow Control}

4.1. Slot Position. The first step of the performance improvement focused on the slot position rather than the profile of the duct because the former had the most significant influence on the intensity of recirculation and the interaction between duct flow and tip flow.

The section view for three configurations of slot position is shown in Figure 8. The conceptual geometry of the casing treatment remained unchanged except for the position of the bleed slot and its corresponding duct wall and guide vanes. The subscripts " $D$ " and " $U$ " represented the fact that the slot was moved $1 / 3 \mathrm{ca}$ downstream/upstream of the baseline position considered in Section 3. The simulated overall performance is plotted in Figure 9. It is demonstrated that the slot position had significant influence on the peak efficiency of the baffled cases but had almost no influence on the nonbaffled cases. As for the flow range, the downstream slot position helped to increase $\Delta m ; \mathrm{CT}_{\mathrm{D}}$ produced higher peak efficiency and larger flow range than the $\mathrm{CT}_{\mathrm{D} \text {,baffled }}$ case.

The variation of efficiency is explained in Figure 10 with the $\Phi_{\text {area }}$ plot at $m=4.2 \mathrm{~kg} / \mathrm{s}$, which corresponds 


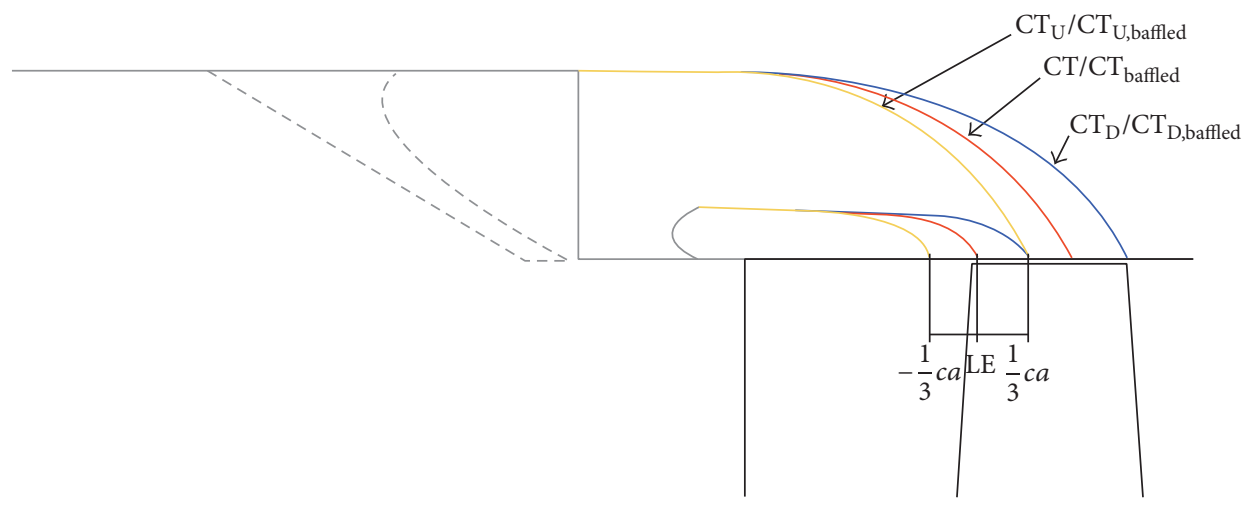

FIGURE 8: Different slot positions.
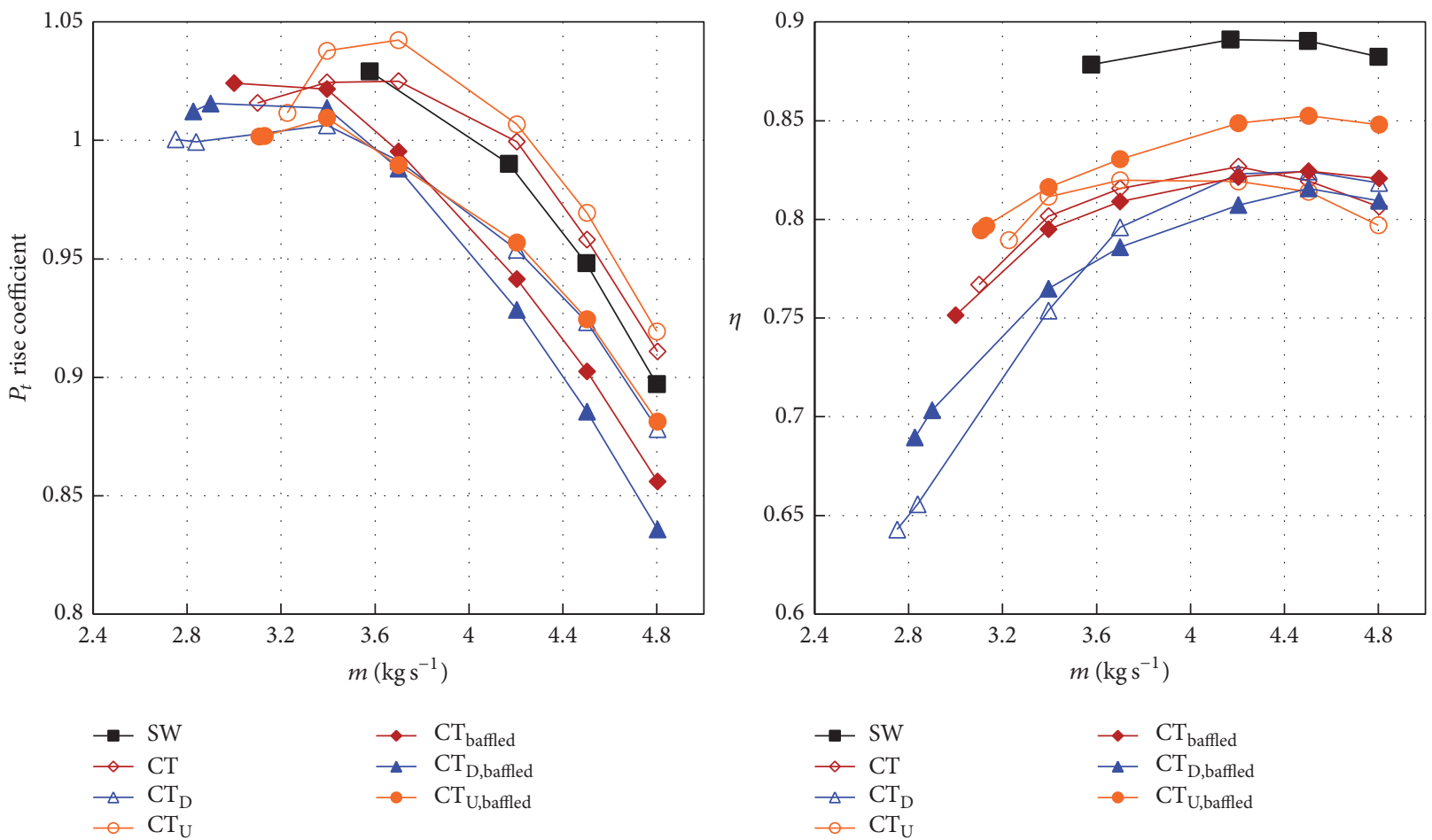

FIgURE 9: Total performance of different slot position.

approximately to the efficiency point. The flow field in tip region is also presented here to illustrate the tip leakage flow (black streamlines), the reverse flow (transparent light green isosurface of $V_{a}=-0.1 \mathrm{U}$ ), and the slot flow (the contour of $\left.V_{r}\right)$. For the nonbaffled casing treatments, the intensity and direction of the flow in the recirculation duct (bleeding or injecting) depended on the position of the slot and where it interacted with the main flow in the blade passage. However, neither $\mathrm{CT}_{\mathrm{D}}$ nor $\mathrm{CT}_{\mathrm{U}}$ showed a satisfactory result. Dissipation from $80 \%$ span to $100 \%$ span made a major contribution to the variation of loss. At the peak efficiency point, $\mathrm{CT}_{U}$ suppressed the leading edge shear flow but induced a strong swirling flow in the rear part of the passage (labeled by the red dashed ellipse). The value of $\Phi_{\text {area }}$ was therefore increased around the downstream edge of slot $(0.7 c a)$ for the $\mathrm{CT}_{\mathrm{U}}$ case and resulted in higher overall loss than for CT. In $\mathrm{CT}_{\mathrm{D}}$, the leakage vortex extended to the upstream region and reached the pressure side of the adjacent blade near the leading edge because of the interaction. The LE shear flow was thus intensified and $\Phi_{\text {area }}$ increased significantly as a consequence. Compared with the nonbaffled cases, the baffled casing treatments all generated recirculation flow in the duct and consequently the distribution of dissipation in the casing treatment represented a higher proportion of the losses. Because of the weaker recirculation flow, $\mathrm{CT}_{\mathrm{U} \text {,baffled }}$ generated the lowest dissipation in the recirculation duct, both in value and range. The peak efficiency of $\mathrm{CT}_{\mathrm{U} \text {,baffled }}$ showed a very notable increase of about $3 \%$.

To explain the difference in flow range, Figure 11 compares the pitch-averaged velocity near the leading edge plane at 

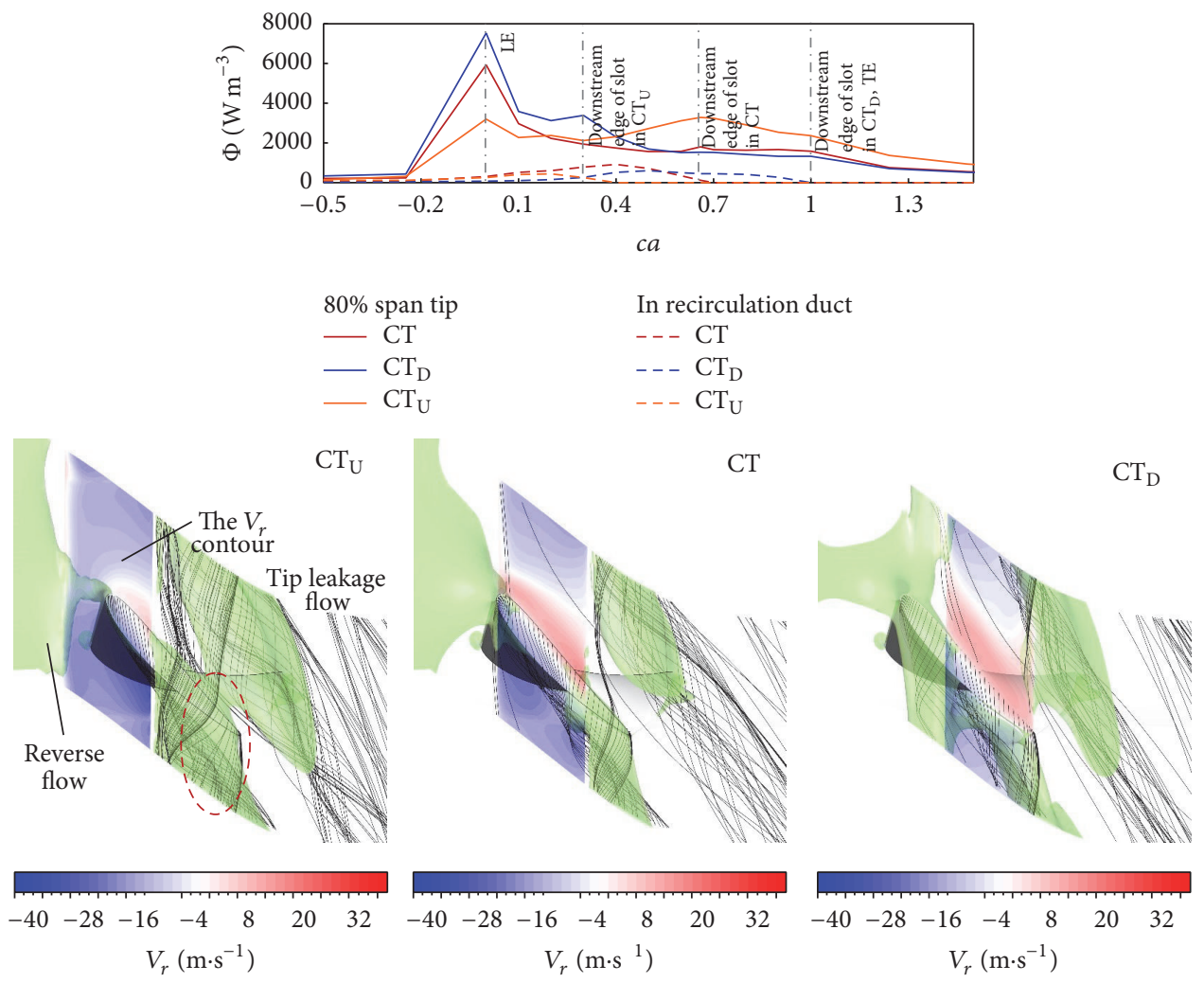

(a) Dissipation function and tip flow field without noise baffle

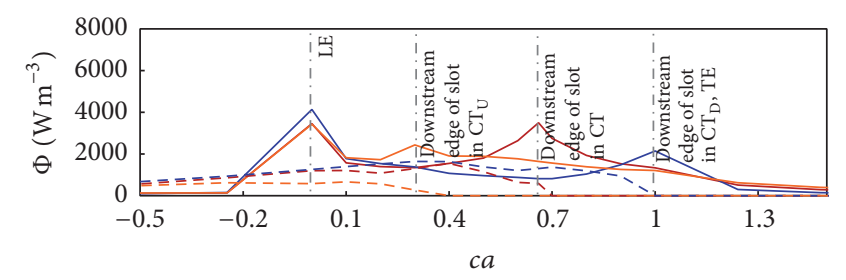

$\begin{array}{ll}80 \% \text { span tip } & \text { In recirculation duct } \\ \text { CT } & ---\mathrm{CT} \\ -\mathrm{CT}_{\mathrm{D}, \text { baffled }} & ---\mathrm{CT}_{\mathrm{D}, \text { baffled }} \\ -\mathrm{CT}_{\mathrm{U}, \text { baffled }} & ---\mathrm{CT}_{\mathrm{U}, \text { baffled }}\end{array}$

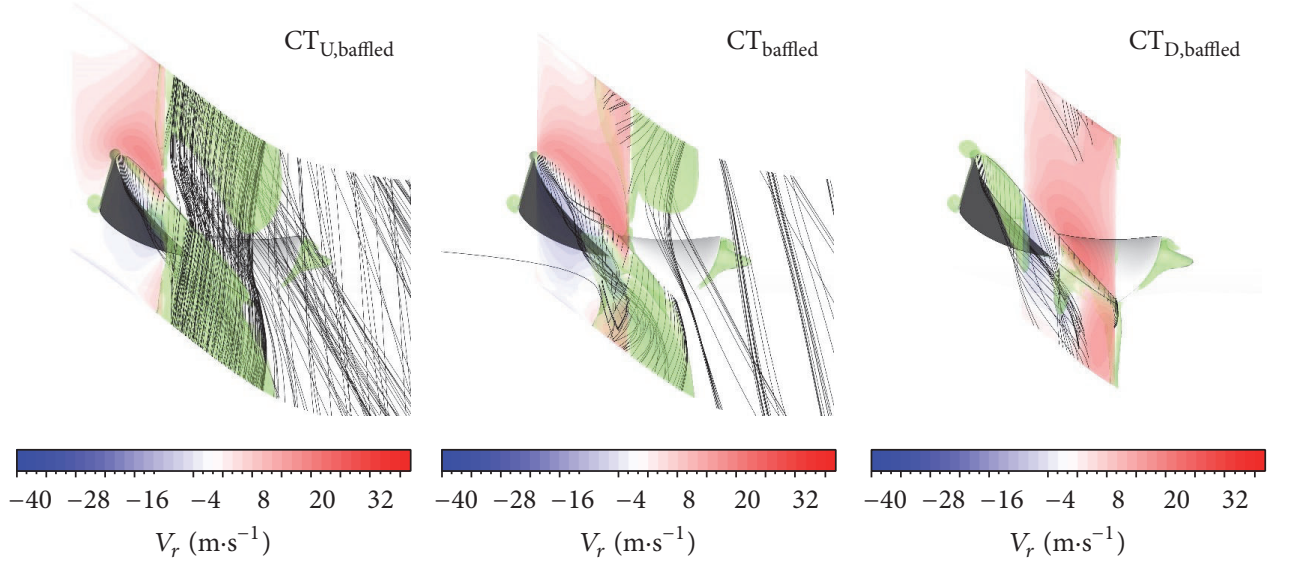

(b) Dissipation function in baffled cases

FIGURE 10: Distribution of loss source in different slot position at $m=4.2 \mathrm{~kg} / \mathrm{s}$. 

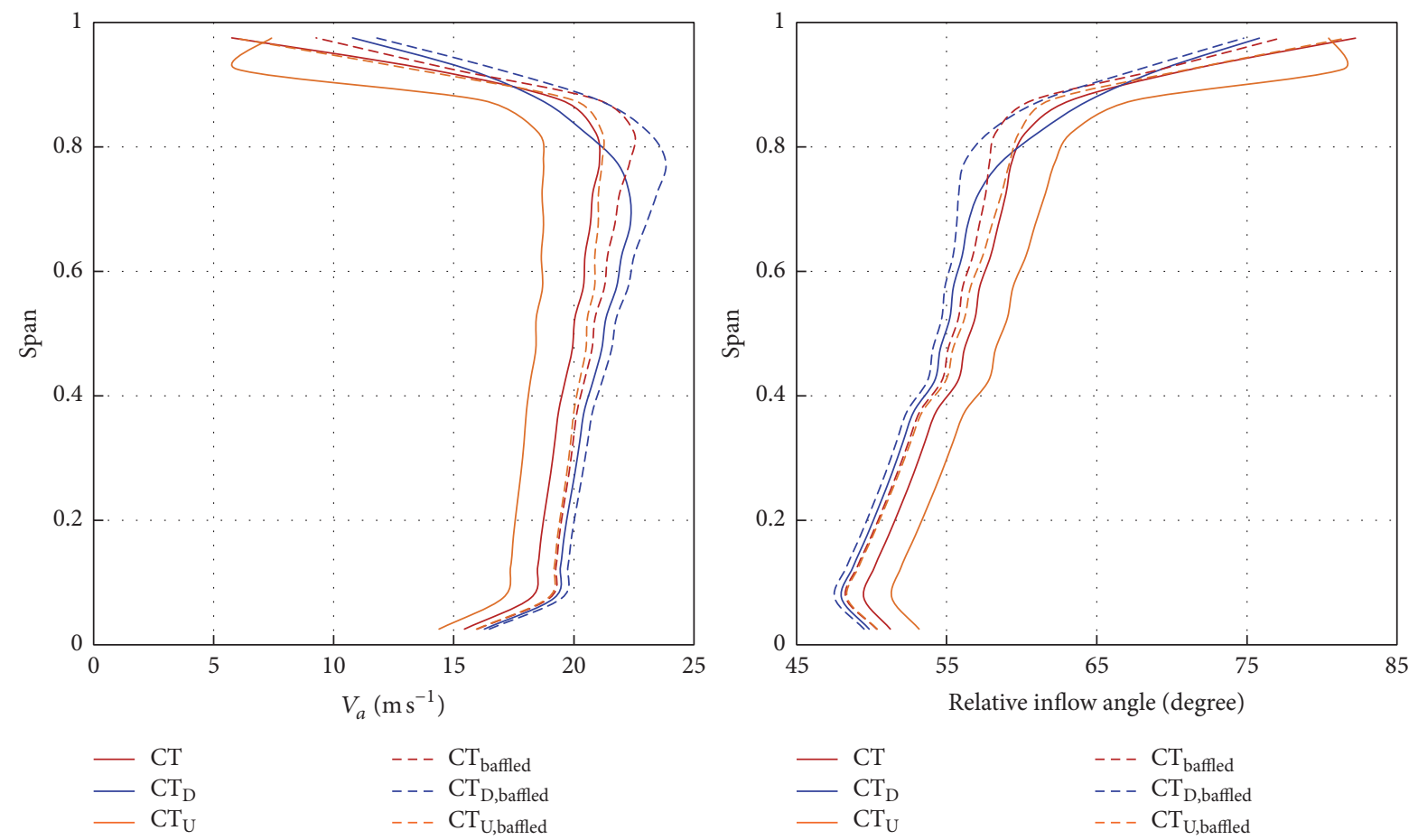

FIGURE 11: Pitch-average velocity near LE at $m=3.4 \mathrm{~kg} / \mathrm{s}$.

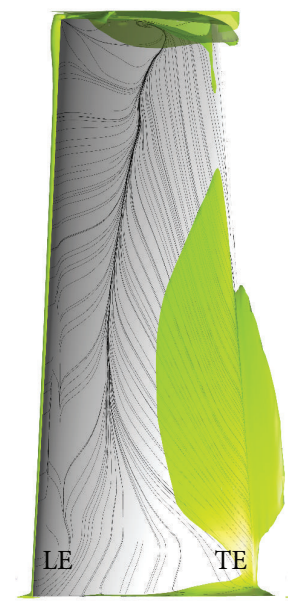

$\mathrm{CT}_{\mathrm{U}, \text { baffled }} m=3.10 \mathrm{~kg} / \mathrm{s} \mathrm{CT}_{\text {baffled }} m=3.0 \mathrm{~kg} / \mathrm{s} \quad \mathrm{CT}_{\mathrm{D}, \text { baffled }} m=2.83 \mathrm{~kg} / \mathrm{s}$

(a)

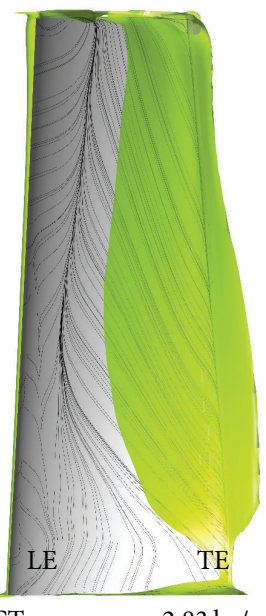

(a)

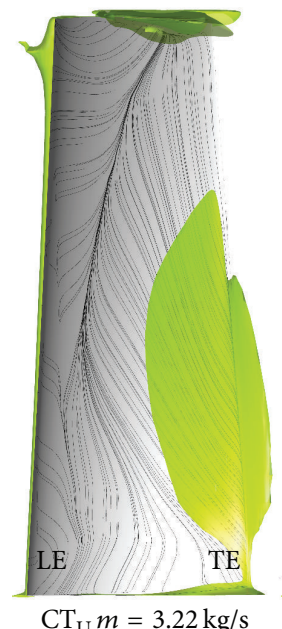

$\mathrm{CT}_{\mathrm{U}} m=3.22 \mathrm{~kg} / \mathrm{s}$

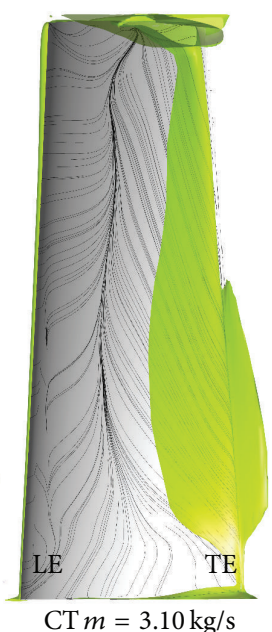

(b)

FIGURE 12: Limiting stream lines and separation on the SS at the NS point of each case.

$m=3.4 \mathrm{~kg} / \mathrm{s}$. Figure 12 gives the flow field of the NS point of each case, in which the isosurface of $V_{a}=-0.1 \mathrm{U}$ is illustrated to show the separation. The following should be noted.

(i) Different intensity of recirculation leads to different span-wise distribution of velocity at the LE. Since the recirculation is guided by the vanes and reinjected into the inflow in a fixed direction in the $t-r$ plane, the higher value of $V_{a}$ is therefore always associated with lower relative flow angle, and thus the lower incidence.

(ii) For the baffled casing treatments, the downstream slot position achieved a lower flow rate at the NS point and therefore produced a larger flow range. At the same mass flow rate of $m=3.4 \mathrm{~kg} / \mathrm{s}$, the downstream slot position resulted in higher $V_{a}$ in the rotor passage (Figure 11), thus indicating a stronger recirculation flow in the duct of casing treatment. 
(iii) The SS flow at the NS point of the baffled cases showed similar characteristics, regardless of their differences in reverse flow region and mass flow rate (Figure 12(a)). The downstream slot position resulted in a more significant separation area in the low-span region of the blade, but it relieved the reverse flow in the tip region at the NS point.

(iv) The nonbaffled casing treatments showed a consistent relationship between the low-span $V_{a}$ and the position of the slot at $m=3.4 \mathrm{~kg} / \mathrm{s}$. However, tip $V_{a}$ did not show a consistent variation as in the baffled cases.

(v) The SS flow at the NS point of nonbaffled cases showed distinct features (Figure 12(b)). But the same correlation between slot position and span-wise separation was found as for the baffled cases.

For the baffled casing treatments, the flow range was limited by the span-wise transport of separation and reverse flow over the full-span of the SS. The recirculation increased as the slot moved downstream. With the same overall mass flow rate, the stronger recirculation would enhance the tip and mid-span axial flow, $V_{a}$. Therefore, the SS separation was relieved because of the lower incidence, which also suppressed the radial transport of low energy separation flow and its accumulation in the tip region. Consequently, the rotor was able to operate stably at a lower mass flow rate, in which the flow distribution at low-span and the tip reverse flow developed to a new level associated with the change of full-span incidence and the intensity of the recirculation. Sheltered and guided by the noise baffle, the upstream extent of the recirculation and the direction of reinjection were similarly restricted, as shown in Figure 12. The reinjected recirculation flow in turn influenced the spanwise distribution of inflow and thus showed a similar flow pattern at the NS point for $\mathrm{CT}_{\text {baffled }}, \mathrm{CT}_{\mathrm{D} \text {, baffled }}$, and $\mathrm{CT}_{\mathrm{U} \text {,baffled }}$.

For the nonbaffled casing treatment, the flow range was also limited by the span-wise transport of separation and reverse flow over the full-span of the SS, the same as the baffled casing treatment. However, the recirculation flow was exposed to the inflow and influenced by both the slot position and the momentum of the upstream flow field. The upstream extent of the recirculation and the direction of reinjection in $z-r$ plane were thus altered depending upon the intensity of the recirculation. With the same overall mass flow rate, the distribution of $V_{a}$ at the tip in relation to the slot position showed different characteristics from the baffled cases. At the NS point, the SS separation of $\mathrm{CT}_{\mathrm{U}}$ showed a similar flow pattern to the baffled cases, while $\mathrm{CT}_{\mathrm{D}}$ tended to reduce the low-span separation more than all the other cases (this can be seen from the area of isosurface or the position of the SS separation line), even though it operated at the lowest overall mass flow rate. It is thus inferred that $\mathrm{CT}_{\mathrm{U}}$ should have the same stall mechanism as the baffled cases, while the more intense recirculation flow in $\mathrm{CT}_{\mathrm{D}}$ can be extended to a lower span without the constraint of the baffle. Since $\mathrm{CT}_{\mathrm{D}}$ achieved the most favorable reduction in the stall flow rate, $\Delta m$, this further extent of the recirculation helped to balance the spanwise distribution of $V_{a}$ better for the flow range improvement.

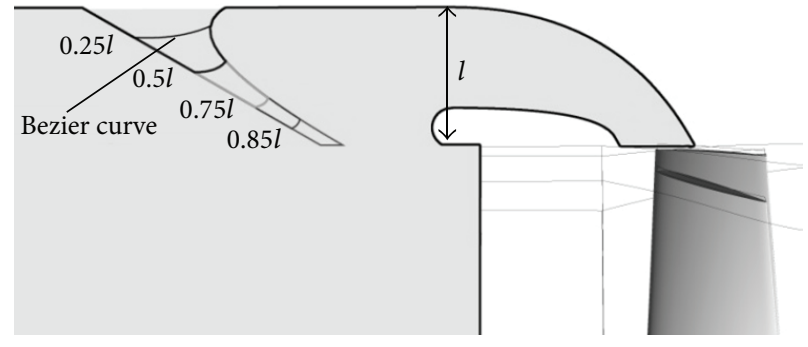

FIGURE 13: The geometry of different noise baffle length.

4.2. Length of the Noise Baffle. Knowing the significance of the noise baffle and its influence in controlling the recirculation flow, the study progressed to focus on the length of the baffle. The main objective was the improvement of peak efficiency, so the upstream casing treatment was used as the baseline case since the highest peak efficiency was achieved with $\mathrm{CT}_{\mathrm{U} \text {,baffled }}$. Figure 13 illustrates the variation of the noise baffle. The length of baffle was measured from the outer wall of the duct to the inner radius of baffle, as shown. A Bezier curve was employed to generate the inner edge of the baffle to achieve a smooth shape for the solid wall. A total of 6 different baffle lengths (including $\mathrm{CT}_{U}$, i.e., zero length) were investigated, as shown in Figure 13.

Figure 14 presents the overall performance of all these cases. The highest peak efficiency was achieved with a baffle length of $0.75 l$, which further improved the peak efficiency of $\mathrm{CT}_{\mathrm{U}}$ by $2.3 \%$ with a slightly higher $\Delta m$ of $17.3 \%$. The baffle of $0.85 l$ had a lower peak efficiency than the baffle of $0.75 l$ but further improved the flow range by $4.0 \%$.

Figure 15 shows the flow field inside the casing treatment duct at $m=4.2 \mathrm{~kg} / \mathrm{s}$. The high dissipation region is shown using the grey isosurface of $\Phi=10^{4} \mathrm{~W} / \mathrm{m}^{3}$ with red curves and blue curves representing the inlet flow and slot flow, respectively. The baffle of $0.75 \mathrm{l}$ achieved the highest peak efficiency because of the balance between inlet flow and the recirculation. The $0.75 \mathrm{l}$ baffle almost stopped the inlet flow from getting into the duct, thus avoiding the high dissipation of the upstream inner ring, as shown in Figure 15(b). On the other hand, the momentum of the inlet flow was strong enough to suppress the development of the slot flow. The intensity of recirculation and the corresponding dissipation of slot flow in the $0.75 l$ case was thus not as significant as for the $0.85 l$ baffle.

It is known from the previous section that the flow range of the $\mathrm{CT}_{\mathrm{U}}$ and $\mathrm{CT}_{\mathrm{U} \text {,baffled }}$ cases was limited by both the spanwise transport of separation and the reverse flow in the fullspan of the SS. The reason for the flow range improvement achieved by different baffle lengths can be explained using the leading edge pitch-averaged velocity at $m=3.4 \mathrm{~kg} / \mathrm{s}$, as shown in Figure 16. Again the higher $V_{a}$ is found to be associated with the lower relative flow angle and thus the lower incidence, just the same as Figure 11. With the upstream slot position, the pitch-averaged $V_{a}$ of the case with the $0.85 l$ baffle was not the largest in full-span range. Below $80 \%$ span, $V_{a}$ of $0.85 l$ baffle was larger than for the $0.25 l, 0.5 l$, and $0.75 l$ baffles but smaller than for the $\mathrm{CT}_{\mathrm{U} \text {,baffled }}$; above $80 \%$ span, 

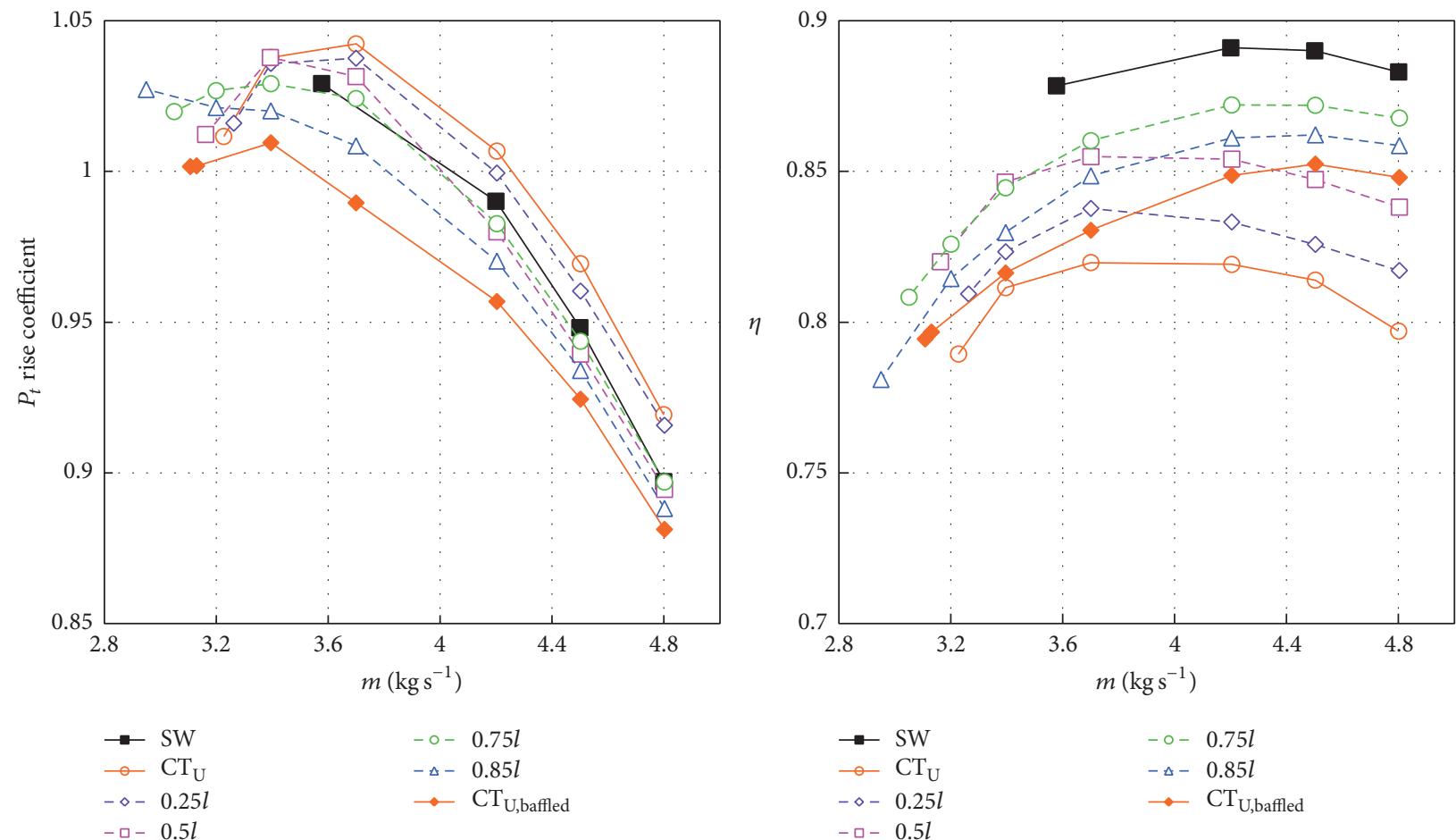

FIgURE 14: Total performance of different baffle length.

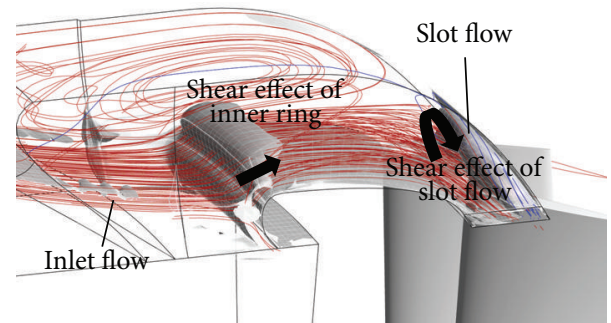

(a) $0.5 l$

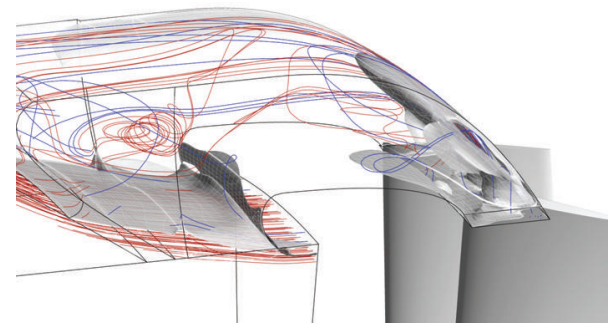

(b) $0.75 l$

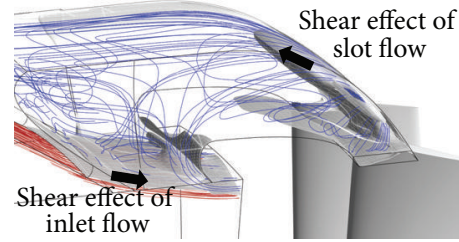

(c) $0.85 l$

FIGURE 15: The dissipation in the recirculation duct.

$V_{a}$ of $0.85 l$ baffle is only larger than for the $0.25 l$ and $\mathrm{CT}_{\mathrm{U}}$. Since the casing treatment with the $0.85 \mathrm{l}$ baffle achieved the largest flow range, it indicates that the $0.85 \mathrm{l}$ baffle has a more balanced control of the tip flow and the low-span inlet flow than all the other cases.

4.3. A Further Flow Range Improvement. The above analysis shows the significance of the redistribution of span-wise velocity on flow range improvement. The influence of slot position and baffle length is summarized and sketched in Figure 17. The analysis is based on the same overall mass flow rate.

For the baffled casing treatments, moving the slot in the downstream direction intensifies the recirculation and thus increases the mass flow rate in the rotor passage over the fullspan range. However, because the reinjected flow in the tip region typically possesses higher axial momentum than the tip blockage but lower than that of the main flow, the increase of axial velocity at about $90 \%$ span is slightly suppressed. As a result, more mass flow rate was concentrated in the tip region and the lower-span region, as shown in Figure 17(a). This explains the distribution of inlet $V_{a}$ for the baffled cases in Figure 11. The influence of baffle length was associated with the intensity of recirculation. For a weak recirculation, the shorter baffle introduced the high-speed inlet flow to the rotor tip and further suppressed the recirculation, as shown by the red arrow and curves. The two effects both helped to decrease the mass flow rate in the lower-span region but 

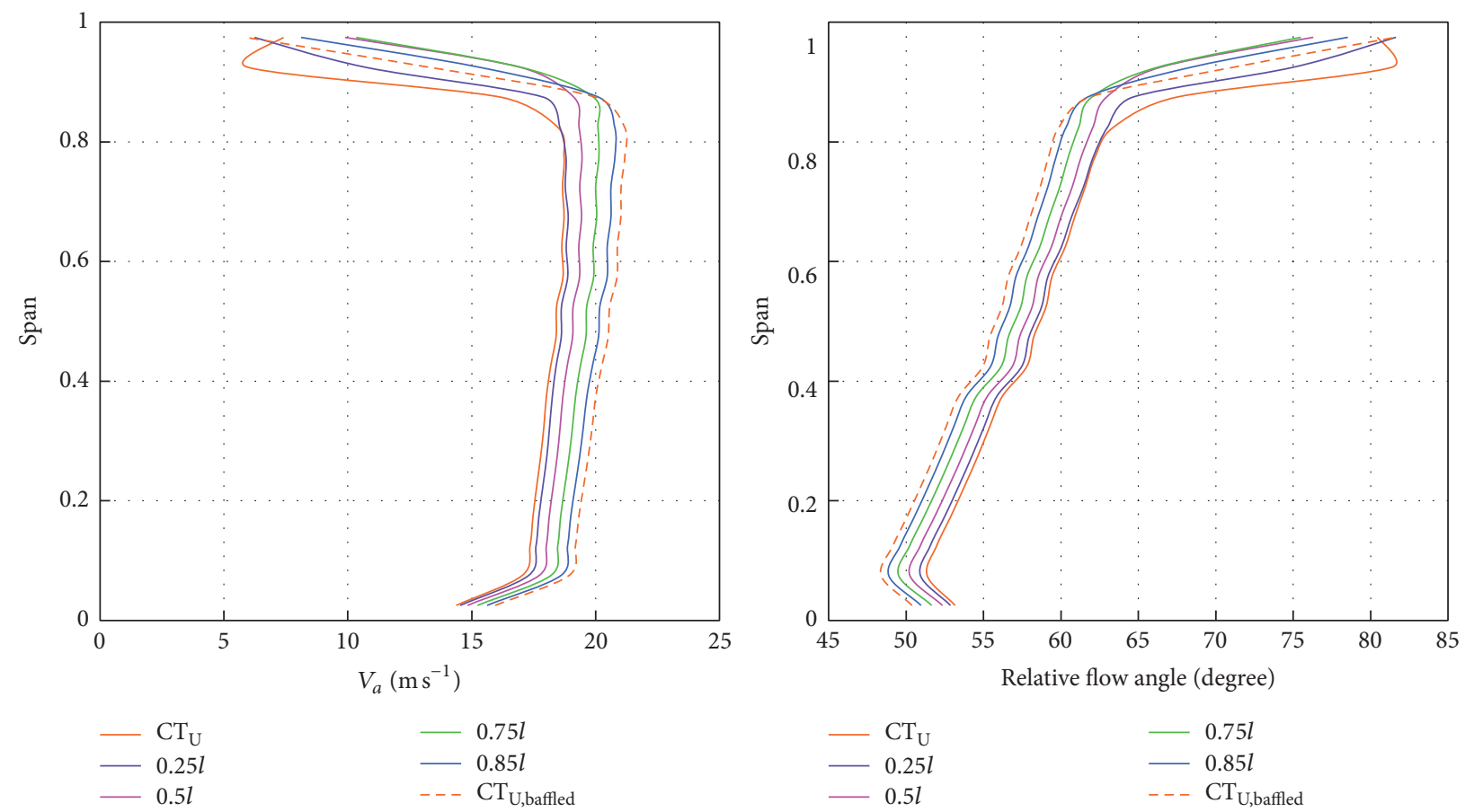

FIGURE 16: The distribution of inlet velocity for different baffle lengths at $m=3.4 \mathrm{~kg} / \mathrm{s}$.

led to differing results in the tip region; the lower-span mass flow rate will be definitely reduced, as shown in Figure 16. For the case with much stronger recirculation, the inlet flow and recirculation were changed in the opposite way, as shown by the blue curve and arrow. The shorter baffle therefore distributed more mass flow rate to the lower-span region.

The different baffle length in Section 4.2 shows a surprising result that flow range can be improved using a suitable length along with higher peak efficiency than $\mathrm{CT}_{U}$ and $\mathrm{CT}_{U, \text { baflled }}$ with almost the same or larger flow range. This section describes the investigation of different baffle lengths for the downstream slot case to see if there is further flow range improvement.

The overall performance is shown in Figure 18. For the downstream slot position, the best baffle length for improved flow range was found to be $0.5 l . \Delta m$ reaching $37.6 \%$ with a peak efficiency of $84.1 \%$. The reason for the slightly improved efficiency compared to $\mathrm{CT}_{\mathrm{D}}$ and $\mathrm{CT}_{\mathrm{D} \text {,baffle }}$ is the same as in Section 4.2 and is not repeated again here. Since the span-wise distribution of $V_{a}$ in Figure 19(a) shows the second type of influence of baffle length defined in Figure 17(b), it is inferred that, at $m=2.83 \mathrm{~kg} / \mathrm{s}$, the casing treatments in all three downstream slot cases generated stronger recirculation than could be suppressed by the inlet flow and $\mathrm{CT}_{\mathrm{D}}$ possessed a much higher level of recirculation than $\mathrm{CT}_{\mathrm{D} \text {,baffled }}$ and the $0.5 \mathrm{l}$ baffle. The flow range achieved with the $0.5 l$ baffle was larger than $\mathrm{CT}_{\mathrm{D} \text {,baffled }}$ because of its higher mid-span axial velocity. According to the discussion in Section 4.1, this relieved the separation and thus suppressed the tip blockage. Compared with the case of the $0.5 l$ baffle, $\mathrm{CT}_{\mathrm{D}}$ showed higher $V_{a}$ over the full-span but achieved a smaller flow range. The reason is shown in Figure 19(b), which shows the entropy at the same U2 station considered in Figure 19(a). $\mathrm{CT}_{\mathrm{D}}$ produced a higher loss above $60 \%$ span at its NS point $(m=2.75 \mathrm{~kg} / \mathrm{s})$. The high entropy was generated by the interaction between the inlet flow and the recirculating flow which extended significantly upstream without the limitation of the baffle. Since the stall flow was judged by a strict criterion which took into account the outlet static pressure rise and high entropy is associated with lower pressure, the reason why $\mathrm{CT}_{\mathrm{D} \text {,bafled }}$ resulted in improved $\Delta m$ compared to $\mathrm{CT}_{\mathrm{D}}$ should lie in its suppression of the upstream extent of the recirculation flow and the corresponding reduced loss near the stall point.

4.4. The Coupled Effect of Baffle Length and the Slot Position on Peak Efficiency and Flow Range. To study the coupled effect of the baffle length and the slot position on the peak efficiency and flow range, more different values of baffle length were simulated to optimize the peak efficiency and the flow range achieved for each slot position. The result was given in Figure 20.

According to Figure 20(a), the downstream slot generally achieved a larger flow range than the upstream cases. The optimum length of the baffle for improving the flow range decreased as the slot moved downstream. In the cases with the downstream slot, the remarkably high value of $m_{\mathrm{NS}}$ adjacent to the optimum $m_{\mathrm{NS}}$ condition indicates that the highspeed recirculation flow due to the downstream slot would reduce the flow range if not suitably controlled. Figure 20(b) shows that the upstream slot generally achieves higher peak efficiency than the downstream slot, and the optimum length of baffle decreases as the slot position moves downstream. 


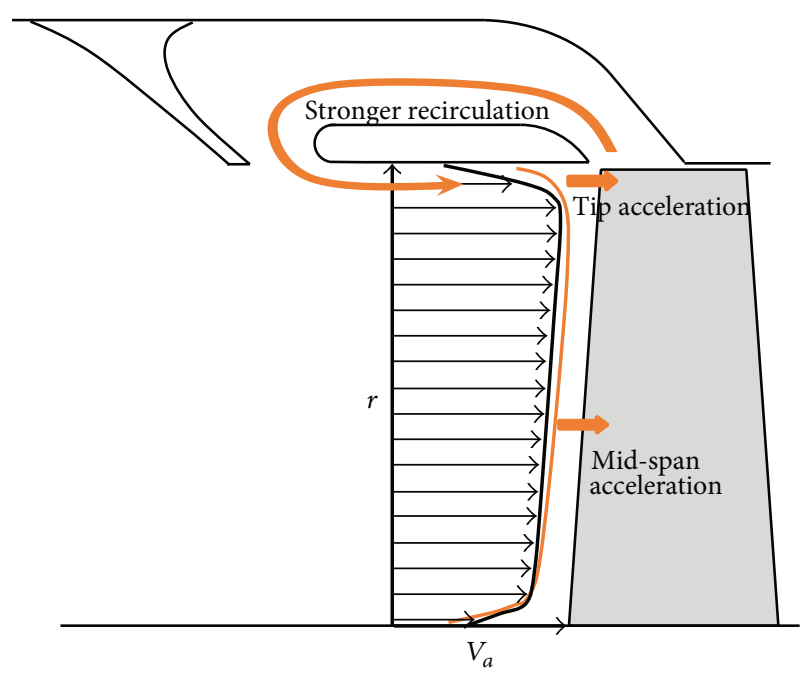

(a) Influence of slot position

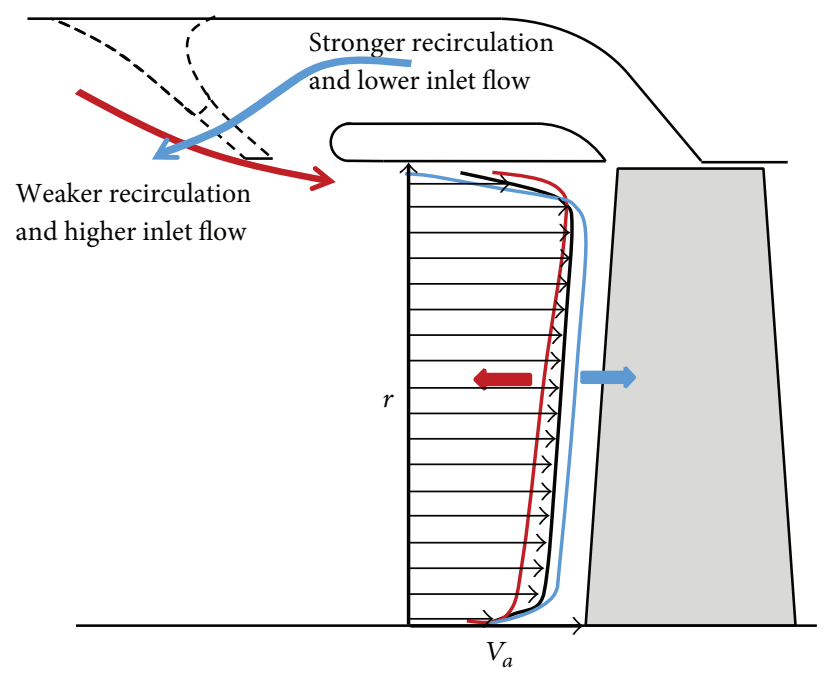

(b) Influence of baffle length

FIGURE 17: The influence of slot position and baffle length on the distribution of inlet velocity.

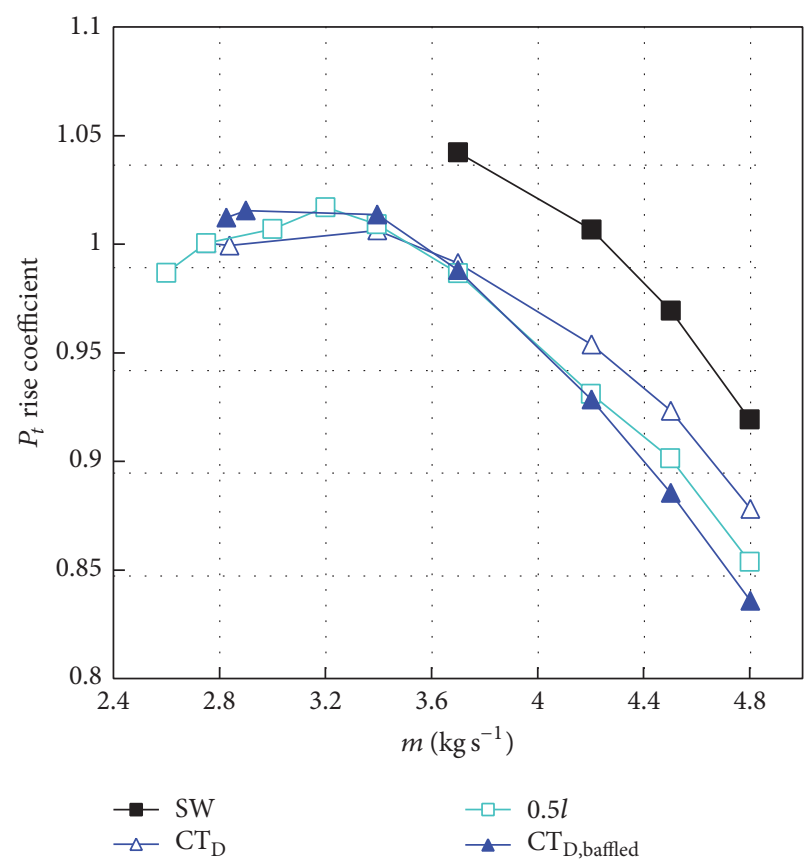

(a)

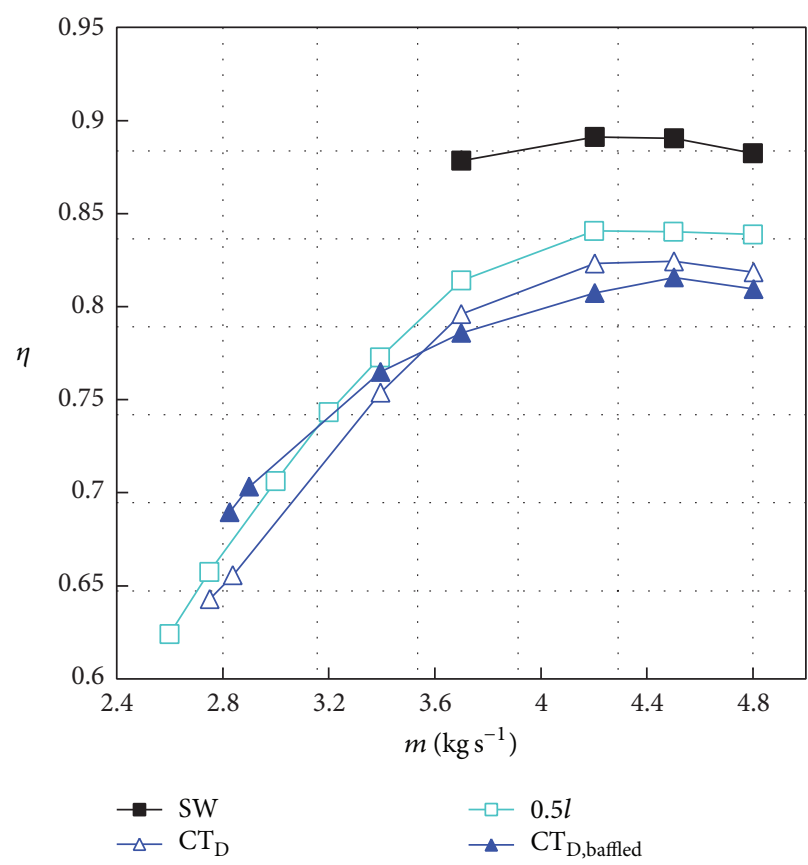

(b)

FIGURE 18: Total performance of downstream slot with different baffle length.

Comparing with the plots of $m_{\mathrm{NS}}$, it can be seen that the value of the optimum baffle length for peak efficiency is always greater than the baffle length for best flow range in each slot position.

\section{Conclusion}

This research applied a counter-swirl self-recirculation casing treatment to a low speed axial fan-rotor to investigate the possibility of reducing the efficiency penalty and improving the stall margin by suitably controlling the duct flow in the casing treatment.

The influence of the slot position and the interaction of the noise baffle was found to be significant for the performance.

For the baffled cases, the duct flow was sheltered and guided by the noise baffle and thus produced recirculation and similar reinjection of flow regardless of the slot position. Moving the slot further downstream increased the intensity of the recirculation. The flow range was thus extended but 


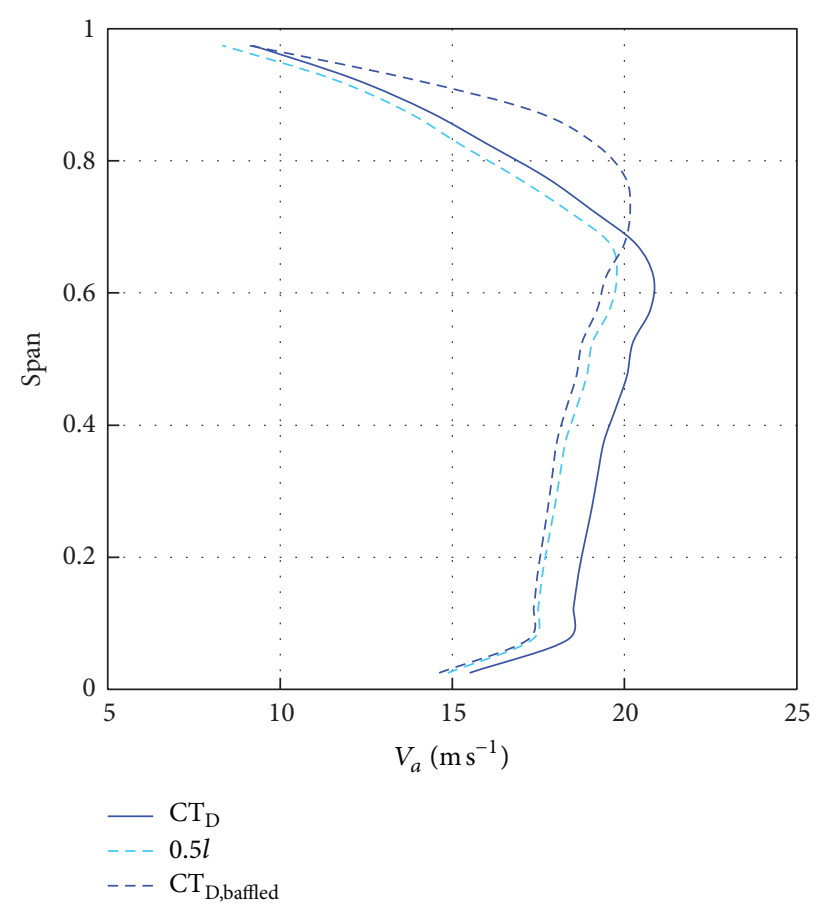

(a) Inlet $V_{a}$ at the NS point of $\mathrm{CT}_{\mathrm{D} \text {, baffled }}(m=2.83 / \mathrm{kg} / \mathrm{s})$

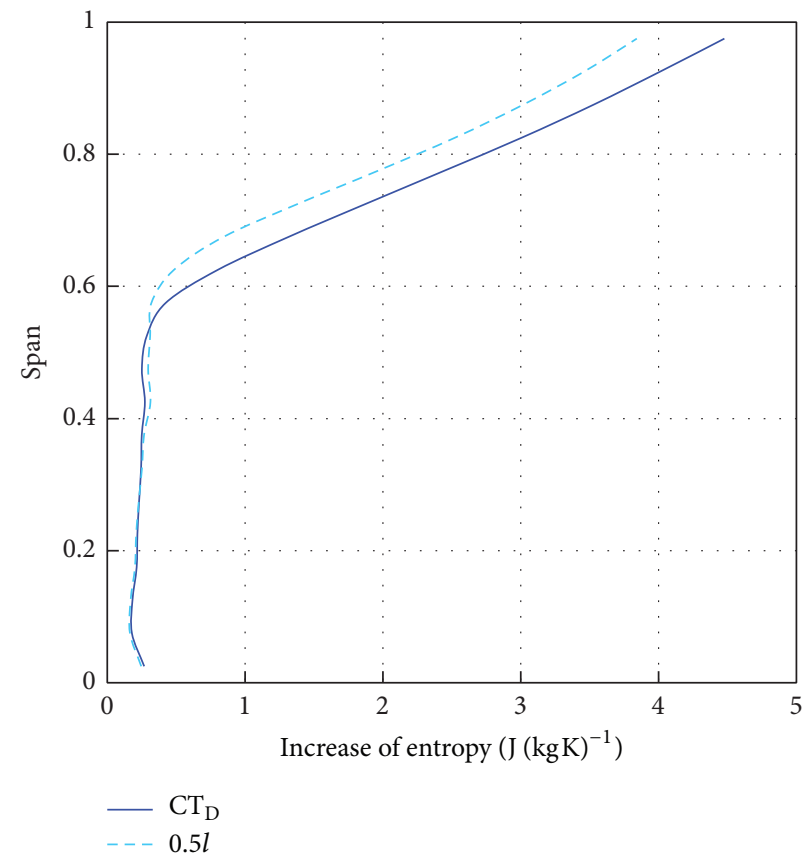

(b) Outlet entropy at the NS point of $\mathrm{CT}_{\mathrm{D}}(m=2.75 / \mathrm{kg} / \mathrm{s})$

Figure 19: Distribution of the pitch-average parameters for $\mathrm{CT}_{\mathrm{D}}, 0.5 l$ baffled case, and $\mathrm{CT}_{\mathrm{D} \text {,baffled }}$.

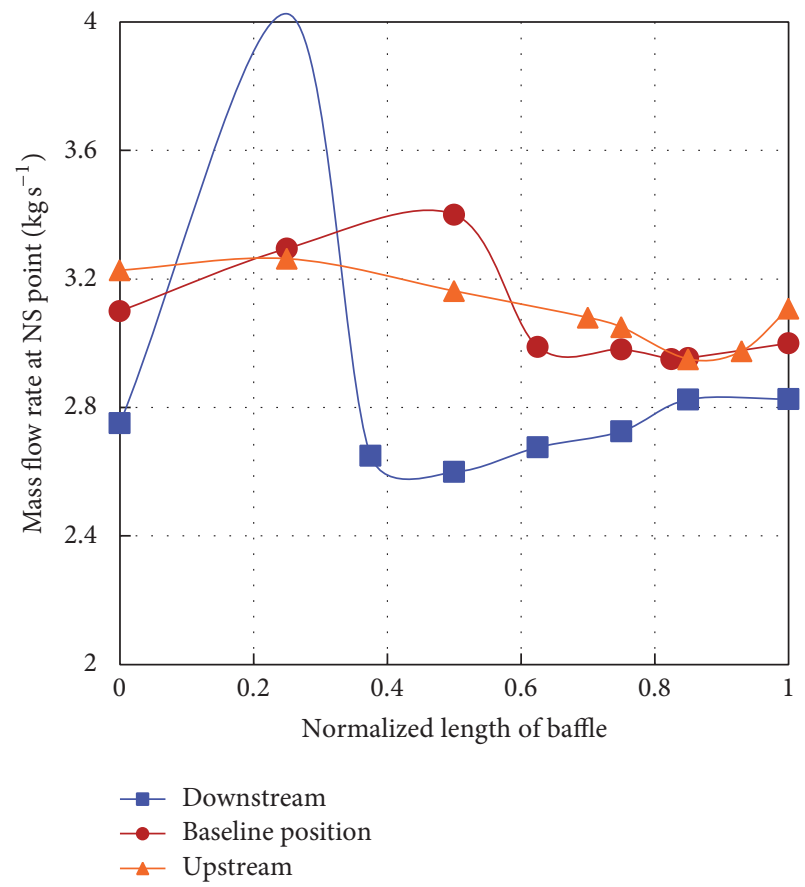

(a)

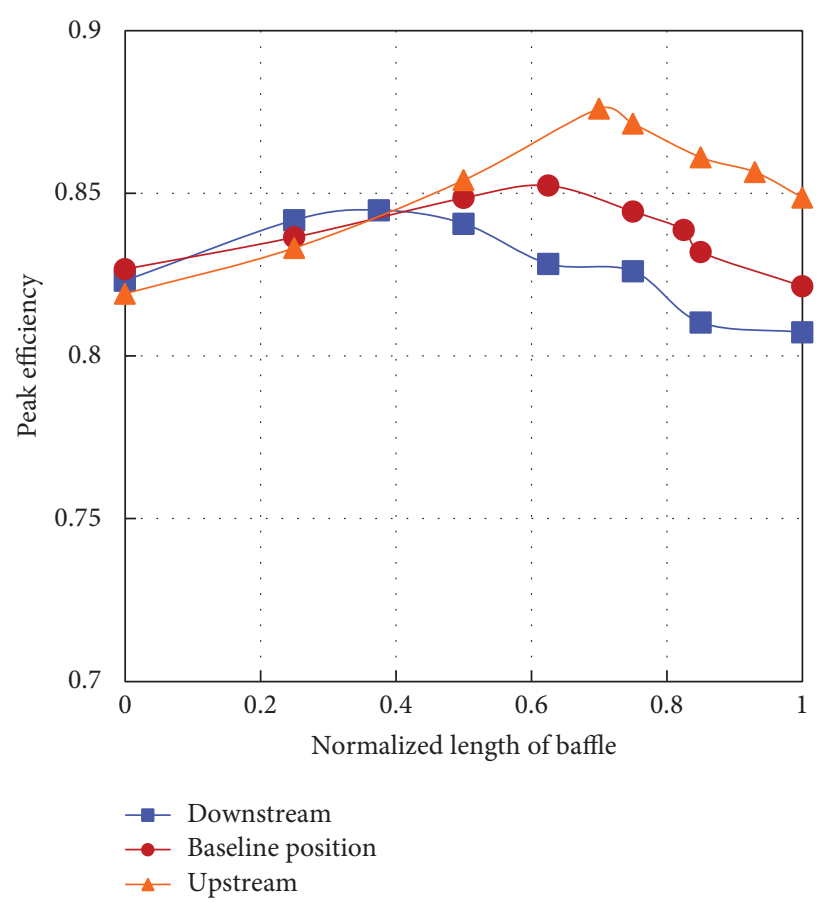

(b)

Figure 20: The coupled effect of slot position and the baffle length on efficiency and flow range. 
incurred more efficiency penalty. The stall condition in all three cases was similarly induced by the accumulation of fullspan separation flow in the tip region of the blade.

For the nonbaffled cases, the direction of the flow in the recirculation duct was found to be associated with the position of the slot. At the peak efficiency point, the slot flow would be in the inward direction when the slot was placed at the upstream location, but flow was bled out of the slot when it was placed at the downstream location; both positions induced additional efficiency penalty. At the NS point, in the absence of the noise baffle, the upstream extent of the recirculation and the direction of reinjection in the $a-r$ plane were altered depending upon the intensity of the recirculation. The downstream slot position showed much larger flow range than the baseline case and the upstream case due to its stronger recirculation.

Knowing the significant influence of the noise baffle, a study of the baffle length was conducted for further performance improvement. Based on the upstream slot position, four more different baffle lengths were simulated and it was found that penalty in peak efficiency was further reduced by $2.3 \%$ (although this was still $1.9 \%$ lower than the smooth casing) by using a part-length baffle of $0.75 l$. In this configuration the inlet flow and the recirculation almost reached a balance point and no strong flow was formed in the recirculation duct at the peak efficiency point. Investigating the effect of baffle length for the downstream slot position showed that, compared with the full-length baffle and the nonbaffled cases, the inlet velocity profile over the full-span was best controlled with the $0.5 \mathrm{l}$ noise baffle. The flow range could be further extended from $30 \%$ to $37.6 \%$ with a relative $1.7 \%$ increase in peak efficiency. The optimum length of baffle is different depending on whether efficiency or flow range is to be maximised. The length of baffle for best efficiency is always greater than for maximum flow range.

\section{Notations}

$\begin{array}{ll}c: & \text { Blade chord } \\ c a: & \text { Axial chord } \\ f_{w}, f_{l}: & \text { Factors to define the change of input of } \\ & \text { work and loss due to employing the casing } \\ & \text { treatment } \\ l: & \text { Length of noise baffle } \\ m: & \text { Mass flow rate } \\ m_{s}: & \text { Mass flow rate near stall point } \\ m_{c}: & \text { Mass flow rate at choke point } \\ P, P_{t}: & \text { Static pressure and total pressure } \\ r: & \text { Radius } \\ s: & \text { Solidity } \\ V: & \text { Velocity } \\ x, y, z: & \text { Cartesian coordinates } \\ \beta 1, \beta 2: & \text { Inlet and outlet angle of camber } \\ \eta: & \text { Isentropic efficiency } \\ \theta: & \text { Camber angle } \\ \xi: & \text { Stagger angle } \\ \pi: & \text { Total pressure ratio } \\ \tau: & \text { Total temperature ratio }\end{array}$

$\phi:$ Mass flow coefficient

$\psi:$ Static to total pressure rise coefficient

$\Phi$ : Dissipation function.

Subscripts

$a, t, r$ : Axial, tangential, and radial

D, U: Upstream and downstream.

\section{Abbreviations}

CT: Casing treatment

SW: Smooth wall casing

NS: Near stall

PS: Pressure side

SS: Suction side

SST: Shear stress transport.

\section{Competing Interests}

The authors declared no potential competing interests with respect to the research, authorship, and/or publication of this article.

\section{Acknowledgments}

This work was funded by National Natural Science Foundation of China (Grant nos. 51576162 and 51536006) and Doctorate Foundation of Northwestern Polytechnical University (CX201519).

\section{References}

[1] R. G. Griffin and L. H. Smith Jr., "Experimental evaluation of outer casing blowing or bleeding of a single stage axial flow compressor, part I -design of rotor blowing and bleeding configurations," NASA CR-54587, NASA, 1966.

[2] M. D. Hathaway, "Passive endwall treatments for enhancing stability," Tech. Rep. NASA/TM-2007-214409, NASA, 2007.

[3] I. Wilke and H.-P. Kau, "A numerical investigation of the flow mechanisms in a high pressure compressor front stage with axial slots," Journal of Turbomachinery, vol. 126, no. 3, pp. 339349, 2004.

[4] A. Shabbir and J. J. Adamczyk, "Flow mechanism for stall margin improvement due to circumferential casing grooves on axial compressors," Journal of Turbomachinery, vol. 127, no. 4, pp. 708-717, 2005.

[5] M. D. Hathaway, "An improved self-recirculating casing treatment concept for enhanced compressor performance," in Proceedings of the 22nd Army Science Conference, Baltimore, Md, USA, December 2000.

[6] M. D. Hathaway, "Self-recirculating casing treatment concept for enhanced compressor performance," in Proceedings of the ASME TURBO EXPO: Turbomachinery, pp. 411-420, Amsterdam, Netherlands, June 2002.

[7] H. Yang, "Unsteady simulation of a transonic compressor coupled with casing treatment," in Proceedings of the 11th Annual Conference of the Computational Fluid Dynamics Society of Canada (CFD '03), Paper No. 75, May 2003. 
[8] H. Yang, D. Neumberger, E. Nicke, and A. Weber, "Numerical investigation of casing treatment mechanisms with a conservative mixed-cell approach," in Proceedings of the ASME Turbo Expo, GT2003-38484, Atlanta, Ga, USA, June 2003.

[9] C. Guinet, J. A. Streit, H.-P. Kau, and V. Gümmer, “Tip gap variation on a transonic rotor in the presence of tip blowing," in Proceedings of the ASME Turbo Expo: Turbine Technical Conference and Exposition (GT '14), Düsseldorf, Germany, June 2014.

[10] C. Guinet, A. Inzenhofer, and V. Gummer, "Influencing parameters of tip blowing interacting with tip flow," in Proceedings of the ASME Turbo Expo, GT2015-42039, 2015.

[11] H. Chen and V.-M. Lei, "Casing treatment and inlet swirl of centrifugal compressors," Journal of Turbomachinery, vol. 135, no. 3, Article ID 041010, 2013.

[12] S. Sivagnanasundaram, S. Spence, J. Early, and B. Nikpour, "An investigation of compressor map width enhancement and the inducer flow field using various configurations of shroud bleed slot," in Proceedings of the ASME Turbo Expo, GT2010-22154, Glasgow, UK, June 2010.

[13] F. B. Fisher, "Application of map width enhancement devices to turbocharger compressor stages," SAE Transactions Journal of Engines, vol. 97, no. 6, 1988.

[14] K. Yelmar and K. Viswanath, "Computational analysis of recess vane geometry modification in the casing treatment approach to enhance stall margin in axial flow fans," in Proceedings of the ASME International Mechanical Engineering Congress and Exposition (IMECE '13), IMECE2013-63096, 2013.

[15] S. C. Kang, A. B. Mckenzie, and R. L. Elder, "Recessed casing treatment effects on fan performance and flow field," in Proceedings of the ASME International Gas Turbine and Aeroengine Congress and Exposition, 95-GT-197, Houston, Tex, USA, June 1995.

[16] K.-J. Choi, J.-H. Kim, and K.-Y. Kim, "Design optimization of circumferential casing grooves for a transonic axial compressor to enhance stall margin," in Proceedings of the ASME Turbo Expo, GT2010-22396, Glasgow, UK, June 2010. 


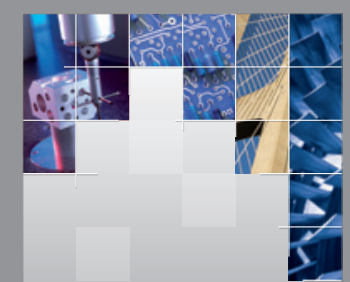

\section{Enfincering}
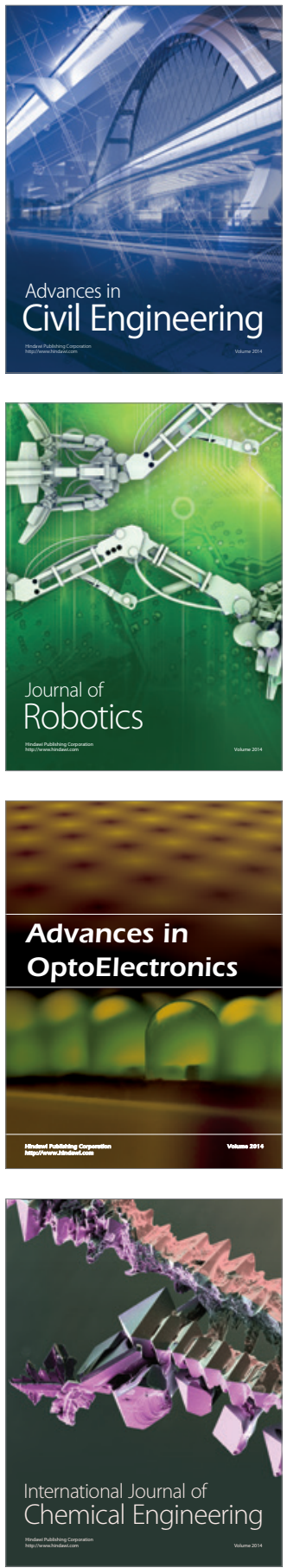

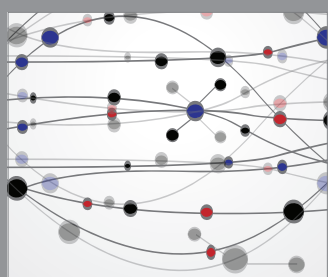

The Scientific World Journal

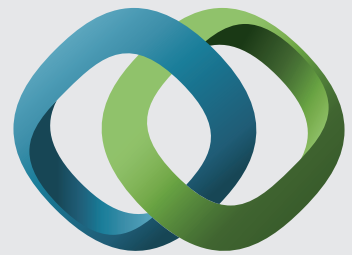

\section{Hindawi}

Submit your manuscripts at

https://www.hindawi.com
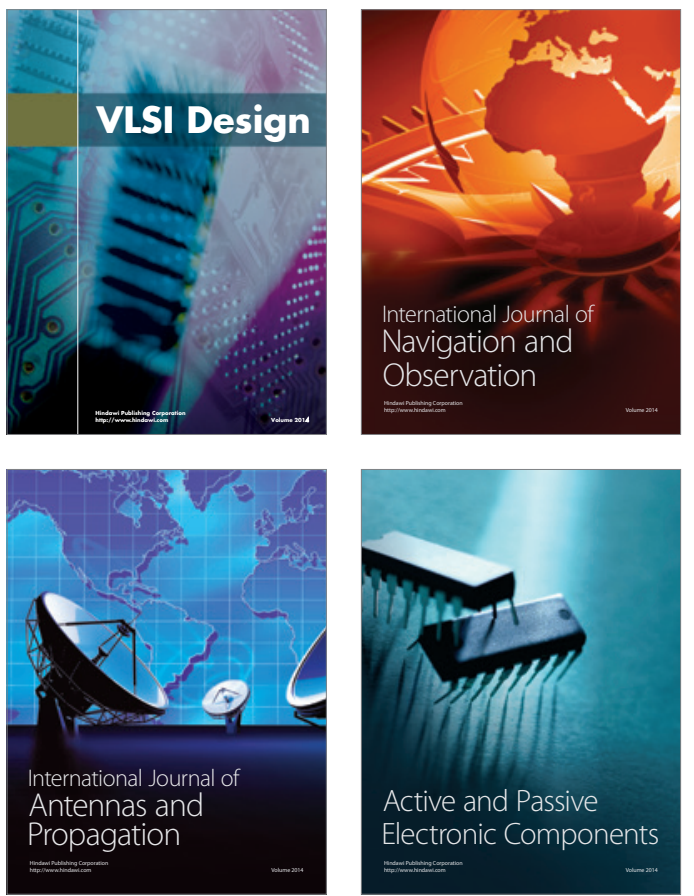
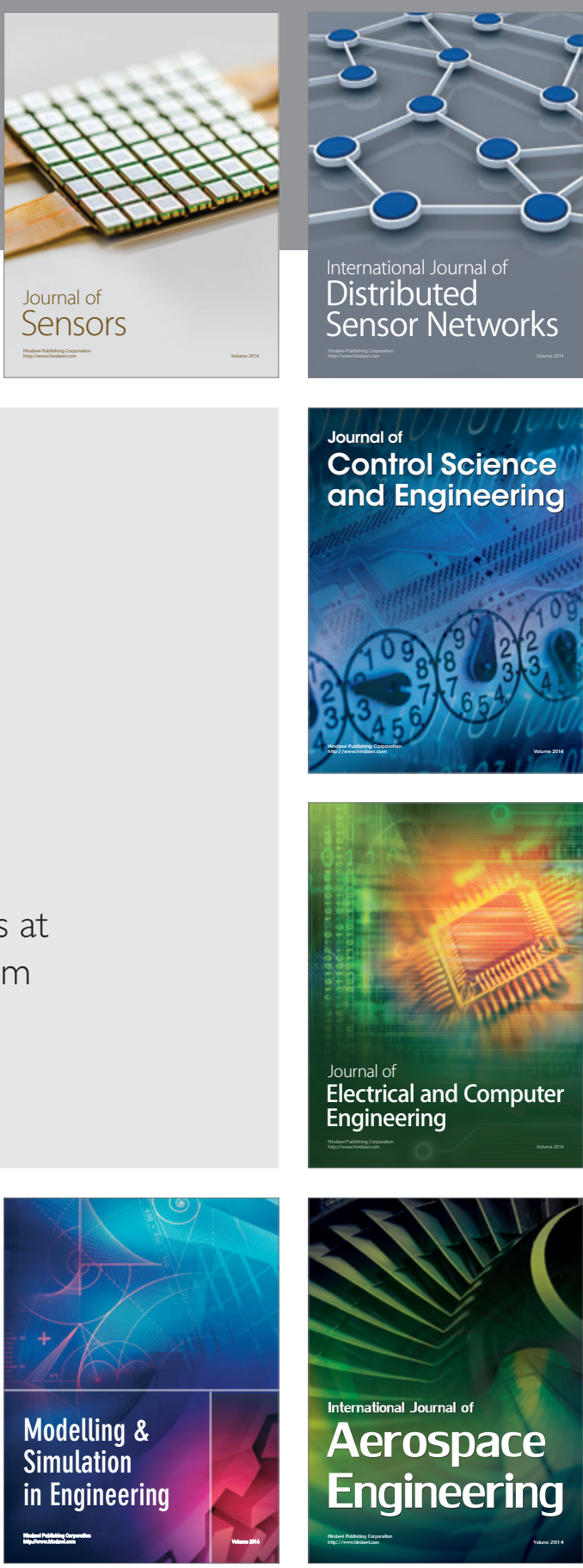

International Journal of

Distributed

Sensor Networks

$-$

Joumal of

Control Science

and Engineering
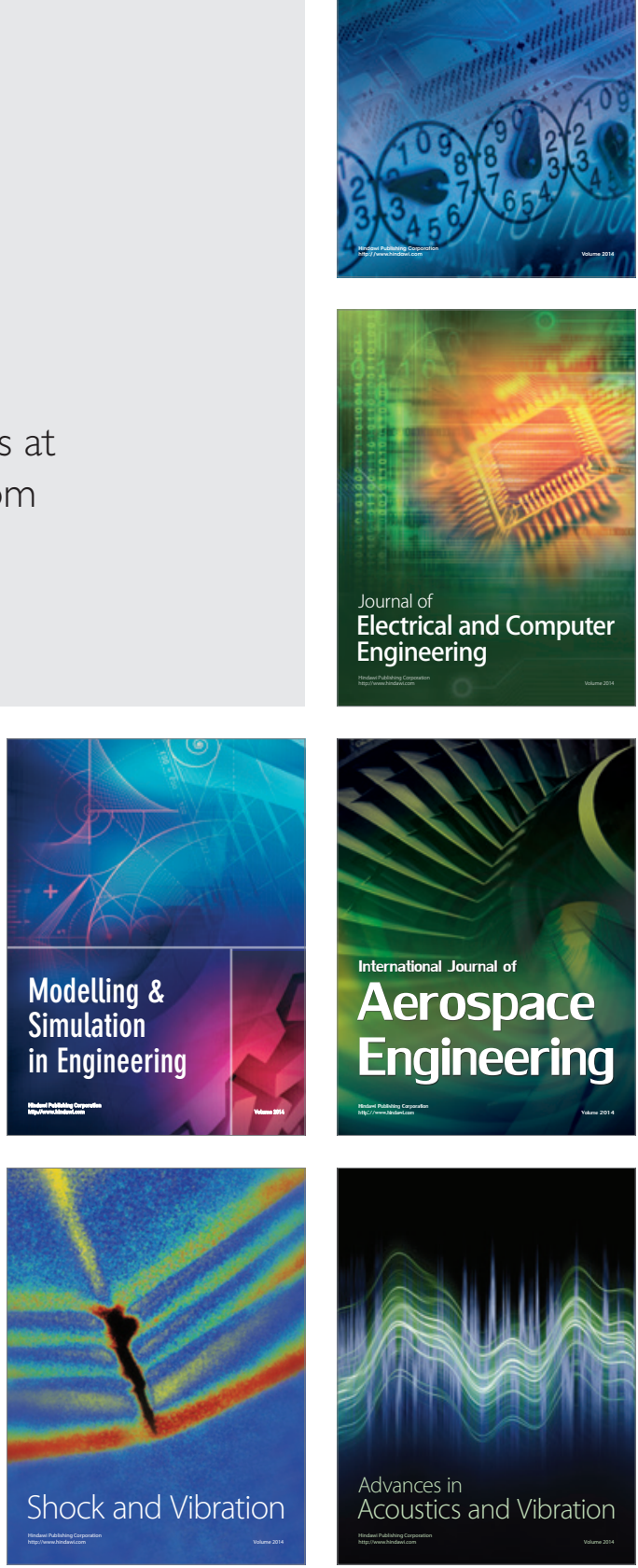